\title{
Coupled atomistic/discrete dislocation method in 3D Part II: Validation of the method
}

\author{
M. Hodapp ${ }^{\mathrm{a}}$, G. Anciaux ${ }^{\mathrm{b}, 1, *}$, J.-F. Molinari ${ }^{\mathrm{b}}$, W.A. Curtin ${ }^{\mathrm{a}}$ \\ ${ }^{a}$ Ecole Polytechnique Fédérale de Lausanne (EPFL) \\ Faculté ENAC-IIC, LSMS (http://lsms.epfl.ch/) \\ CH-1015 Lausanne, Switzerland \\ ${ }^{b}$ Ecole Polytechnique Fédérale de Lausanne (EPFL) \\ Faculté STI, LAMMM (http://lammm.epfl.ch/) \\ CH-1015 Lausanne, Switzerland
}

\begin{abstract}
A methodology for coupling a fully atomistic domain to a surrounding domain described by discrete dislocation plasticity, including the treatment of hybrid dislocation lines that span between the two domains, was presented in the first paper of this series [2]. Here, key features of the methodology are assessed quantitatively within a quasi-static framework at $0 \mathrm{~K}$. To avoid solving an expensive but standard complementary problem for the atomistic/continuum coupling of mechanical fields, which is not essential to the key features of the method, a simplified model for obtaining accurate stress and displacement fields is introduced and validated. The test problem consists of the bow-out of a single dislocation in a semi-periodic box under an applied shear stress, and excellent results are obtained in comparison to fully-atomistic solutions of the same problem.
\end{abstract}

Keywords: Multiscale modeling, Plasticity, Model reduction, Atomistic/continuum coupling, Dislocations

\section{Introduction}

Predictive mathematical tools for modeling plasticity at different length scales began to develop in the beginning of the last century. The current theoretical frameworks can be broadly grouped into atomic, meso, micro- and macroscale models, describing phenomena across scales from individual dislocation motion in nanometer specimens to accumulated plastic flow in large structures. Since plasticity is now established to be size-dependent, and since important controlling dislocation phenomena occur at the atomic scale, the understanding of macroscopic behavior can often require handling plasticity phenomena across several scales simultaneously.

The discrete dislocation dynamics (DDD) method has been developed to study metal plasticity at the micron scale by following the collective motion of complex dislocation arrays. The DDD method must be informed by rules/laws regarding dislocation mobility, dislocation reactions, and interactions of dislocations with metallurgical defects. These latter phenomena are atomistic in nature, and atomistic simulations can be used to provide the necessary input in many simple cases. However, dislocation nucleation and interactions with defects (surfaces, crack tips, voids, solutes, grain boundaries) involves inherently atomistic response that can be difficult to characterize at the level of discrete dislocation line defects. It is not possible, however, to treat mesoscale plasticity problems using purely atomistic methods, due to the computational cost. Thus, it is beneficial to have a methodology that directly couples evolving atomistic and discrete dislocation domains, such that atomistic behavior beyond the capabilities of the DDD method can be modeled explicitly in local regions, outside of which the plasticity evolves via the DDD rules. Atomistic/continuum coupling

${ }^{*}$ Corresponding author

${ }^{1}$ E-mail address: guillaume.anciaux@epfl.ch 
of mechanical fields wherein all inelastic phenomena are contained only in the atomistic domain has been achieved in many methods, starting with the Quasicontinuum method [27] and continue to evolve today $[14,29,23,15,1]$. Extending the methods to handle dislocation plasticity in the continuum domain, and with nearly seamless passing of dislocations back and forth between atomistic and continuum domains, was achieved in the 2d plane strain limit by the Coupled Atomistic and Discrete Dislocations (CADD) method $[21,22,16]$. In $2 \mathrm{~d}$, where the dislocation line direction is perpendicular to the plane of analysis, the individual dislocations are wholly contained within the atomistic domain or the DDD domain. While $3 \mathrm{~d}$ methods can also handle dislocations solely in both domains, there has been no method for dealing with the full problem wherein individual dislocation lines exist in both domains simultaneously, so-called hybrid dislocations. Since many dislocation phenomena occur in 3d, the development of a full 3d CADD method provides powerful new capability for realistic multiscale simulation of dislocation plasticity.

In a companion paper [2], we have proposed such a 3d CADD method, denoted as CADD-3d. The transition region of a hybrid dislocation between atomistic and continuum-line representations is accomplished through a template imposed at the atomistic/continuum interface that enriches the continuum-line description with an atomistic description of the dislocation core structure. This template thus approximates the atomistic environment that the atomistic system would have if embedded in a fully atomistic domain, and thus minimizes coupling errors at the crucial core region of the dislocation as the line passes from one description to the other. An algorithm for the coupled evolution of such hybrid dislocations was also presented in detail. This enables the atomic region to experience accurate forces from the dislocation(s) spanning both domains.

In the present work, we aim to quantitatively assess this key concept of CADD-3d. More precisely, we are interested in the error in the evolution of the hybrid dislocation line, induced by the boundary condition on the atomistic problem which is enriched with the dislocation core template. Therefore we have selected a relatively simple quasi-static test problem which focuses on the main aspects by neglecting non-essential parts such as dynamics and external boundary conditions. One feature of fully $3 \mathrm{~d}$ problems is the necessity of solving for the stress fields throughout the entire domain. This is normally accomplished using a computationally expensive finite-element solution to solve the complementary problem for the $\hat{\bullet}$ fields, as stated in Paper I. To avoid this part of the method for the time being, since this aspect of the problem is well-established conceptually, we introduce a simplified methodology that is valid in infinite domains and when the atomistic domain only contains dislocations (no other defects). In this limit, we compute the entire problem as if it is purely a DDD problem for all dislocation segments, including those in the atomistic domain, and compute the necessary stress fields on the true DDD segments and displacement fields on the atomistic boundary analytically via the $\tilde{\bullet}$ fields plus the superimposed stress field at infinity. We validate this simplified methodology independently of our CADD-3d test problem. We then apply the CADD-3d method to study the periodic bow-out of a single dislocation, for which reference solutions in essentially infinite domains can be obtained for both fully atomistic and fully DDD problems. This test problem allows us to isolate the modeling of the evolution of a hybrid dislocation and demonstrate minimal errors relative to the reference fully atomistic solution.

The remainder of this paper is organized as follows. In Section 2 we first state the governing equations of CADD-3d in a general quasi-static setting at $0 \mathrm{~K}$. Subsequently the approximate problem is derived from the general formulation. We present a practical yet efficient alternating Schwarz-type solution procedure and highlight the advantages of the approximate formulation but also show its limitations by quantifying spurious effects on dislocations in the atomistic domain depending on the complexity of the linear elastic solution used to compute the dislocation fields of the atomic dislocation. In Section 3 we present the numerical validation of the method and discuss key features.

\section{Coupled atomistic/discrete dislocations in $3 \mathrm{D}$}

\subsection{General formulation}

In paper I [2], we have presented the general algorithm for the coupling of an atomistic domain $\Omega_{\mathrm{A}}$ to a continuum domain $\Omega_{\mathrm{C}}$ described by discrete dislocation dynamics, for systems containing dislocations spanning both domains (hybrid dislocations formed by the intersection of the two lines $\gamma_{\mathrm{a}}$ and $\gamma_{\mathrm{c}}$ as shown in Figure 1). The (artificial) interface between both subdomains is denoted as $\partial \Omega_{\mathrm{I}}$. At any instant or increment of 
loading, the atomistic problem involves the interactions of atoms via interatomic potentials subjected to boundary conditions on a surrounding atomistic pad region $\Omega_{\mathrm{P}} \subset \Omega_{\mathrm{C}}$ whose atomic positions are dictated by the solution of the continuum dislocations problem. The associated continuum problem involves a smallstrain elasticity solution of a discrete dislocation dynamics problem subjected to the displacement boundary conditions associated with atomic positions at the atomistic/continuum interface plus any boundary conditions applied on the external boundaries. For hybrid dislocations, the continuum displacement field in the pad region is enriched by the addition of a corrective displacement field $\Delta \tilde{\boldsymbol{u}}_{\text {corr }}\left(\boldsymbol{x} ; \boldsymbol{b}, \vartheta_{\mathrm{p}}\right)$ that approximates the true atomistic core structure of the hybrid dislocation at the interface with character angle $\vartheta_{\mathrm{p}}$ and Burgers vector $\boldsymbol{b}$. An algorithm for the simultaneous evolution of both atomistic and continuum domains was then presented for the case of full quasi-dynamic coupling (quasi-static evolution of the dislocation dynamics problem).

In that work, and here, we do not address the creation of hybrid dislocations by the impingement of dislocations onto the interface region, whether originating from the atomistic or continuum domains. We focus on the evolution of hybrid dislocations to demonstrate that this crucial feature of CADD-3d leads to a very accurate representation of the true system evolution.

The method requires the identification of atomistic portions of the hybrid dislocations near the atom/continuum interface. An atomistic dislocation is a discrete atomic configuration, not a continuous line. Thus, to identify a continuous line representation of an atomistic dislocation, we use a simplified variant of the Dislocation Extraction Algorithm (DXA) by Stukowski et al. [24] as described in Paper I [2, Appendix C, Algorithm 2].

With the above schematic background of the method, we can more carefully define the solution of the quasistatic coupled problem as the fields $\boldsymbol{u}_{\mathrm{a}}, \boldsymbol{u}_{\mathrm{c}}$ and $\gamma_{\mathrm{c}}$ that solve

$$
\mathcal{P}^{\text {cadd }}\left\{\begin{array}{rlr}
\frac{\text { Atomistic problem } \mathcal{P}^{\mathrm{a}}:}{\boldsymbol{f}_{\mathrm{a}}=\mathbf{0}} & \\
\boldsymbol{u}_{\mathrm{a}}=\boldsymbol{u}_{\mathrm{c}}+\Delta \tilde{\boldsymbol{u}}_{\mathrm{corr}} & & \text { in } \Omega_{\mathrm{P}}, \\
\frac{\text { DDD problem } \mathcal{P}^{\mathrm{c} / \mathrm{dd}}:}{\operatorname{div}(\boldsymbol{\sigma})}=\mathbf{0} & & \\
\boldsymbol{u}_{\mathrm{c}}=\boldsymbol{u}_{\mathrm{a}}-\Delta \tilde{\boldsymbol{u}}_{\mathrm{corr}} & & \text { on } \partial \Omega_{\mathrm{I}}, \\
\boldsymbol{f}^{\mathrm{pk}}+\boldsymbol{f}^{\mathrm{core}}=\mathbf{0} & \text { on } \gamma_{\mathrm{c}}, \\
\gamma_{\mathrm{c}}=\gamma_{\mathrm{a}} & \text { on } \partial \Omega_{\mathrm{I}},
\end{array}\right.
$$

in the absence of body forces. Note that we have omitted the natural boundary conditions (displacements/tractions) on the outer boundary $\partial \Omega_{\mathrm{C}} \backslash \partial \Omega_{\mathrm{I}}$ for compactness.

In order to solve problem $\mathcal{P}^{\text {cadd }}$ numerically, we adapt Algorithm 1 from [2] into an implicit scheme which iterates between both subproblems. This approach, designated as Algorithm 1, yields a classical multiplica-

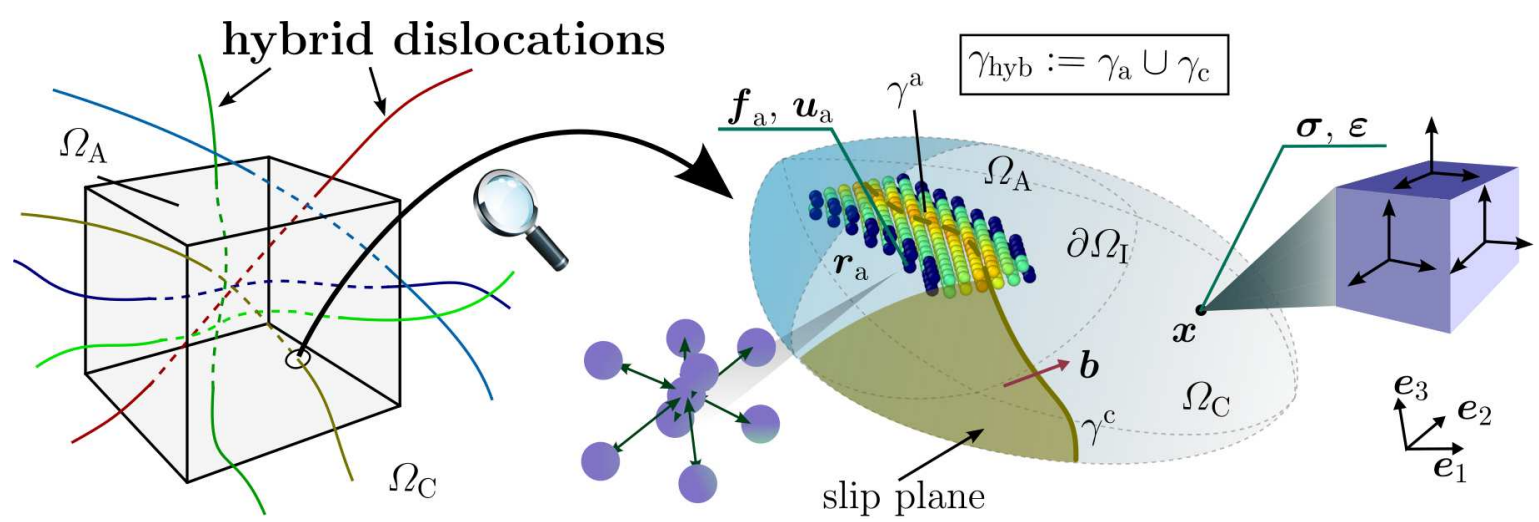

Figure 1: Schematic illustration of the boundary value problem for CADD-3d 
Algorithm 1: Multiplicative alternating Schwarz-type algorithm for CADD-3d

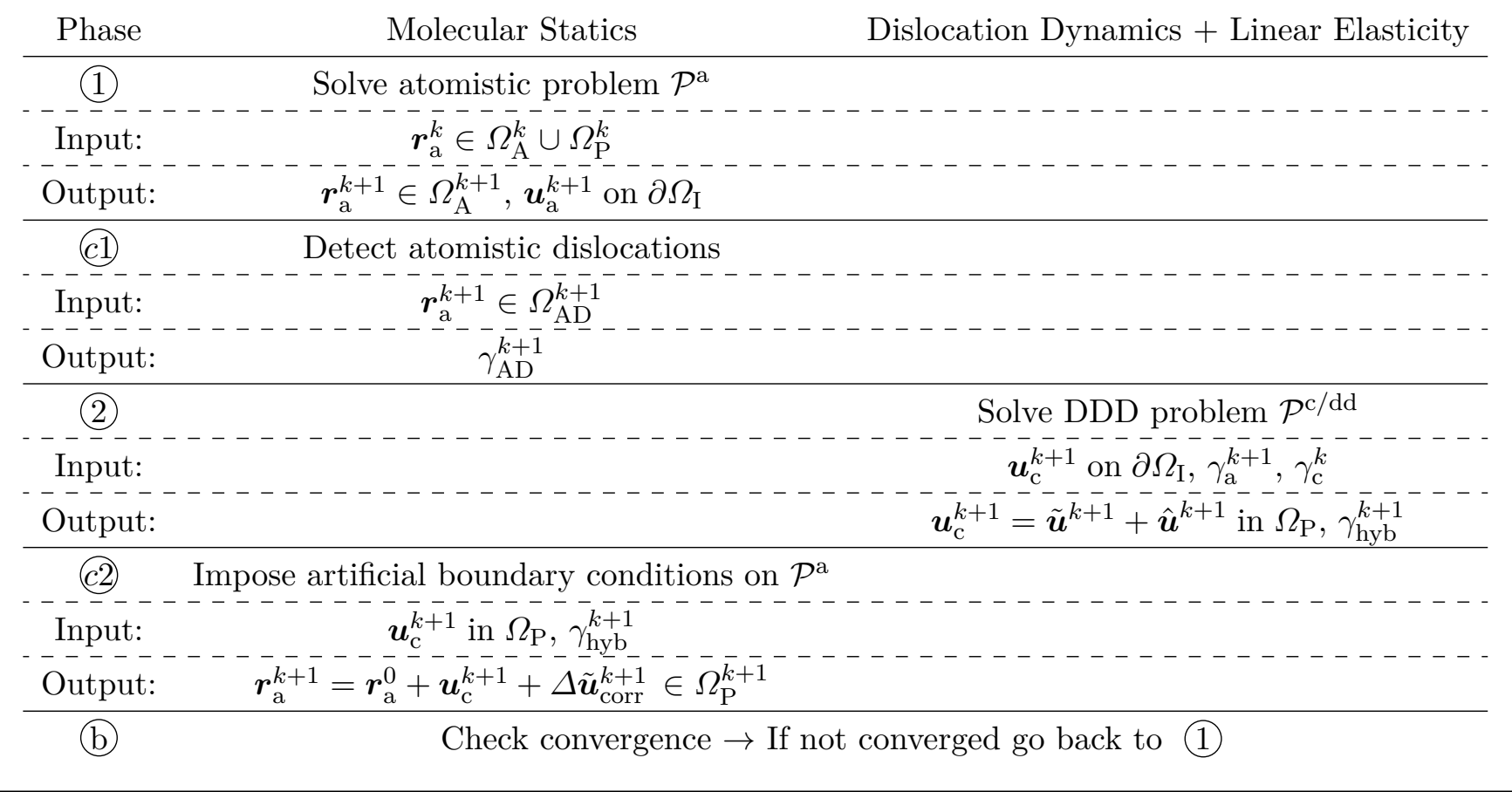

tive alternating Schwarz scheme with sharp interface coupling. In this approach, we essentially iterate between two subproblems. Thus, we replace the 4 phases from [2] with two phases (1) and (2) which correspond to the subproblems defined in (1). Consequently, the time step is replaced by a global iteration. Quantities at a global iteration $k$ are denoted as $\bullet^{k}$. In phase (1) we fully solve the atomistic problem up to a prescribed tolerance using the initial configuration of the previous iteration as input. For the case $k=1$ we impose an initial guess, e.g. based on the linear elastic solution for a given configuration of the dislocation network plus the core correction in the vicinity of the dislocation line. Next, we re-detect the dislocation near the artificial interface in the coupling phase (c1) (this region was termed $\Omega_{\mathrm{AD}}$ in paper I). The detected part $\gamma_{\mathrm{AD}}^{k+1}$ is then connected to the remaining parts in $\Omega_{\mathrm{A}} \backslash \Omega_{\mathrm{AD}}$ and $\Omega_{\mathrm{C}}$ as shown in paper I such that $\gamma_{\mathrm{a}}^{k+1} \cup \gamma_{\mathrm{c}}^{k}$ forms a closed loop. Subsequently the problem $\mathcal{P}^{\mathrm{c} / \mathrm{dd}}$ is fully solved in phase (2) for the continuum displacements $\boldsymbol{u}_{\mathrm{c}}$ and the dislocation line $\gamma_{\mathrm{c}}$. Using the elastic solution in the pad $\boldsymbol{u}_{\mathrm{c}}^{k+1}$ and the location of the hybrid dislocation $\gamma_{\text {hyb }}^{k+1}:=\gamma_{\mathrm{a}}^{k+1} \cup \gamma_{\mathrm{c}}^{k+1}$ we update the pad atoms and the core correction where $\gamma_{\text {hyb }}^{k+1}$ intersects the artificial interface according to (c2). The algorithm terminates for some suitably chosen convergence criterion in (b) , e.g. incremental differences between two iterates of the solution.

However, we still lack a numerical solver coupled with the DDD problem in order to solve for the elastic correction problem (the $\hat{\bullet}$ fields). Therefore we will develop an approximate algorithm in the following section that suffices to test the crucial feature of CADD-3d, namely the boundary condition $\boldsymbol{u}_{\mathrm{a}}=\boldsymbol{u}_{\mathrm{c}}+\Delta \tilde{\boldsymbol{u}}_{\text {corr }}$ in $\Omega_{\mathrm{P}}$, and does not require an external FE solver.

\subsection{Approximate formulation for quasi-static CADD-3d}

In Paper I, we discuss the full coupled boundary value problem, which is solved by a domain decomposition method (c.f. Figure 2 (a)). The DDD problem is then solved by the well-established superposition method wherein all fields are decomposed into a sum of the $\tilde{\bullet}$ fields $(\tilde{\boldsymbol{u}}, \tilde{\boldsymbol{\sigma}})$ of the dislocation network embedded in an infinite homogeneous elastic continuum under zero load plus the $\hat{\boldsymbol{\theta}}$ fields $(\hat{\boldsymbol{u}}, \hat{\boldsymbol{\sigma}})$ arising from an associated smooth corrective problem so that the sum satisfies equilibrium and all boundary conditions in the continuum domain. The choice of $\gamma_{\mathrm{v}}$, which is used to close the dislocation loop in $\Omega_{\mathrm{A}}$, is then arbitrary to some degree (c.f. [2]). The solution for the $\hat{\boldsymbol{u}}$ fields is numerical, and normally accomplished using the finite element method (FEM), which in full $3 \mathrm{~d}$ is computational very expensive. Solving the $3 \mathrm{~d}$ FEM problem is conceptually straightforward and has been implemented by Weygand et al. [28]. Crone et al. [8] 
have also accounted for finite tractions, neglecting $\tilde{\boldsymbol{u}}$ on the boundary. Neither implementation is available as an open-source code, however.

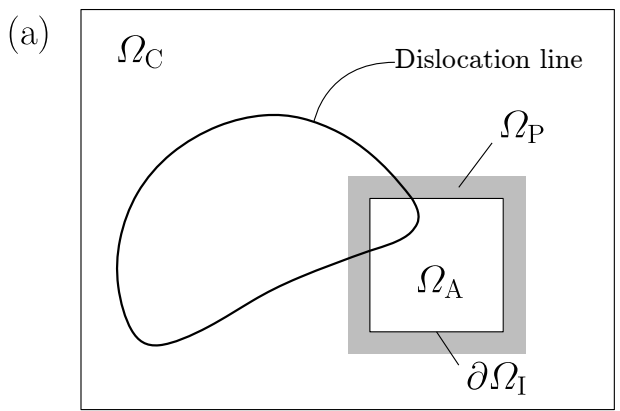

(b)
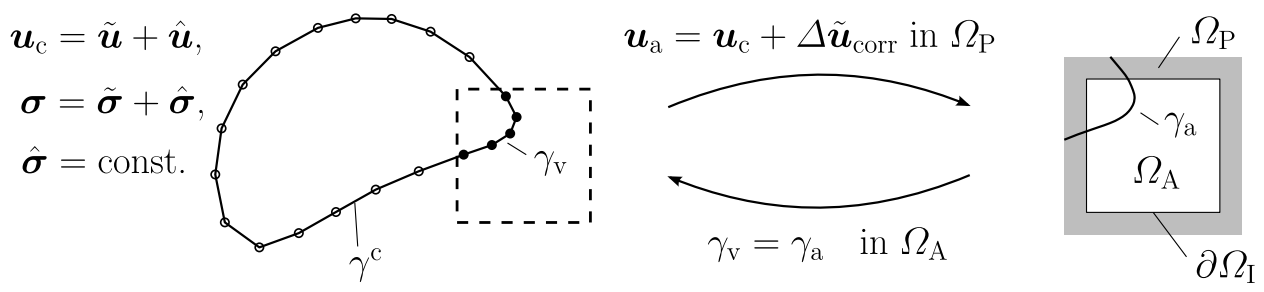

Figure 2: (a) Schematic of the general CADD-3d problem from [2]. (b) Approximate solution of $\tilde{\mathcal{P}}^{\text {cadd }}$ (2) by decomposition into an infinite space DDD problem using the detected atomistic dislocation positions and an atomistic problem with boundary conditions supplied by the displacement fields computed from the infinite space DDD problem.

In the present paper, our aim is to test crucial features of the CADD-3d coupling of hybrid dislocations. This can be done using the problems involving only dislocations and atoms embedded in an infinite homogeneous elastic domain and subjected to a far-field applied stress state. Such problems still involve the crucial mechanical coupling at the atomistic/continuum interface. To eliminate the need to compute the corrective - fields due to the atomistic/continuum coupling, we proceed as shown schematically in Figure 2 (b). We first restrict attention to problems wherein the atomistic domain only contains dislocations (no other defects) and we detect the atomistic dislocation lines $\left(\gamma_{a}\right)$ throughout the entire atomistic domain. This is not an approximation, merely a characterization of the entire problem in terms of the existing dislocation network. We then use the entire dislocation network $\gamma_{\text {hyb }}:=\gamma_{c} \cup \gamma_{a}$ to define a fully-continuum DDD problem within the infinite continuum elastic domain $\Omega_{c} \cup \Omega_{a} \equiv \mathbb{R}^{3}$; that is, we set $\gamma_{\mathrm{v}}=\gamma_{\mathrm{a}}$ (Figure 2 (b)). The solution of this DDD problem is thus the analytic $\boldsymbol{\bullet}$ fields plus the analytic $\hat{\bullet}$ fields due to the constant remote applied stress. There are no other corrective $\hat{\bullet}$ fields. From the DDD displacement field $\tilde{\boldsymbol{u}}+\hat{\boldsymbol{u}}$, we then compute the displacement fields of the pad atoms in $\Omega_{p}$ and include the template correction field for the hybrid dislocations. These displacements then serve, as in the full problem, as the boundary conditions for the atomistic problem. In other words, the boundary conditions for the atomistic problem are obtained from the elastic solution of the entire dislocation network including the dislocation lines inside the atomistic domain. The incremental evolution of the coupled problem (motion of the DDD nodes in $\Omega_{c}$ and motion of the atoms in $\Omega_{a}$ ) is then as described in Paper I and, for quasistatic equilibrium problems, as described below.

In the following, physical quantities $\bullet^{0}$ and $\bullet$ such as the displacement of the body will refer to the initial and deformed states, respectively; we will omit the superscript for the initial state if not explicitly required. 
Otherwise, we adopt the notation of [2]. The reduced problem can be stated as

$$
\tilde{\mathcal{P}}^{\text {cadd }}\left\{\begin{array}{cc}
\frac{\text { Atomistic problem } \mathcal{P}^{\mathrm{a}}:}{\boldsymbol{f}_{\mathrm{a}}=\mathbf{0}} & \text { in } \Omega_{\mathrm{A}}, \\
\boldsymbol{u}_{\mathrm{a}}=\boldsymbol{u}_{\mathrm{c}}+\Delta \tilde{\boldsymbol{u}}_{\mathrm{corr}} & \text { in } \Omega_{\mathrm{P}}, \\
\frac{\text { DDD problem } \mathcal{P}^{\mathrm{c} / \mathrm{dd}}:}{\operatorname{div}(\boldsymbol{\sigma})=\mathbf{0}} & \text { in } \mathbb{R}^{3}, \\
\boldsymbol{f}^{\mathrm{pk}}+\boldsymbol{f}^{\mathrm{core}}=\mathbf{0} & \text { on } \gamma_{\mathrm{c}}, \\
\gamma_{\mathrm{v}}=\gamma_{\mathrm{a}} & \text { in } \Omega_{\mathrm{A}} .
\end{array}\right.
$$

To solve (2) numerically for quasistatic equilibrium problems, Algorithm 1 can be simplified considerably. Following Algorithm 2 we replace the dislocation detection in $\Omega_{\mathrm{AD}}$ (Algorithm 1, phase (c1)) with a detection in the entire atomistic domain in (c1) followed by an equilibration of the discrete dislocation segments only (phase (2)). Otherwise Algorithm 1 remains unchanged. ${ }^{2}$

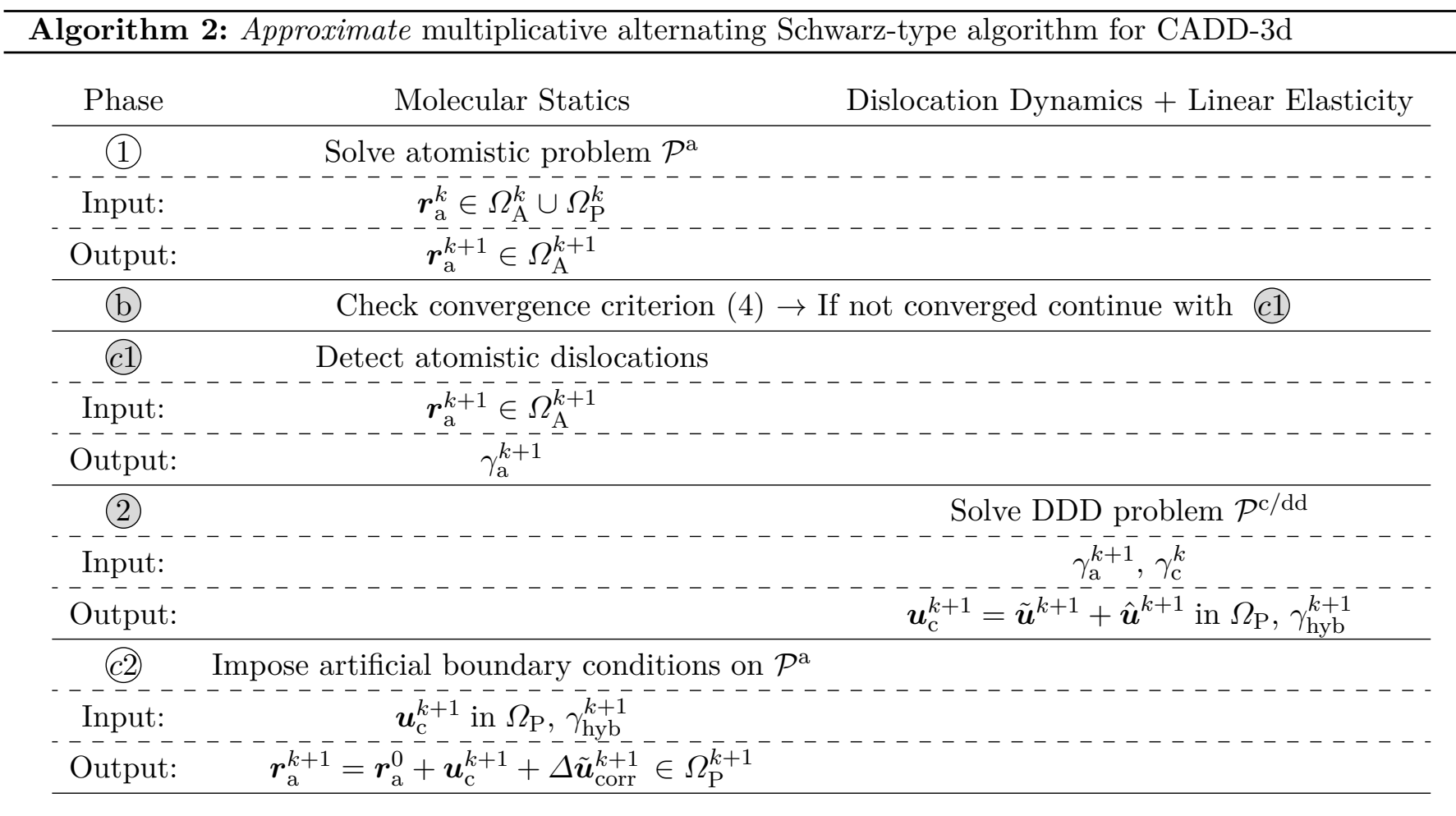

To make the entire solution procedure precise we first select an initial configuration for the entire system. A schematic illustration is shown in Figure 3 (a) for a dislocation segment in the vicinity of the interface spanning both domains. This initial configuration is specified by the displacements of both real atoms in $\Omega_{\mathrm{A}}^{0}$ and pad atoms in $\Omega_{\mathrm{P}}^{0}$ as well as the nodal positions of the discrete hybrid dislocation(s). We then solve the defined atomistic problem $\mathcal{P}^{\mathrm{a}}$ in phase (1) such that the dislocation in the atomistic domain evolves as shown in Figure 3 (b). Using the new atomistic positions, a new dislocation may be detected based on some convergence condition in (b) which will be specified in the following paragraph. Assume for now that a new dislocation line is detected in the atomistic domain and the hybrid dislocation line is updated in (c1). Subsequently the discrete dislocation problem $\mathcal{P}^{\mathrm{c} / \mathrm{dd}}$ is solved in (2) to evolve the dislocation nodes residing in the continuum domain, as shown in Figure 3 (c). With the new continuum dislocation

\footnotetext{
${ }^{2}$ In order to highlight the modified parts we have grayed out the corresponding phase indicators
} 
line, the pad atoms are then updated using the new displacement field of the entire dislocation network in (c2). The core template solution is imposed for hybrid dislocations using the character angle defined by the straight segment crossing the pad region (c.f. Figure 3 (c)). The above steps are performed iteratively until convergence is obtained.

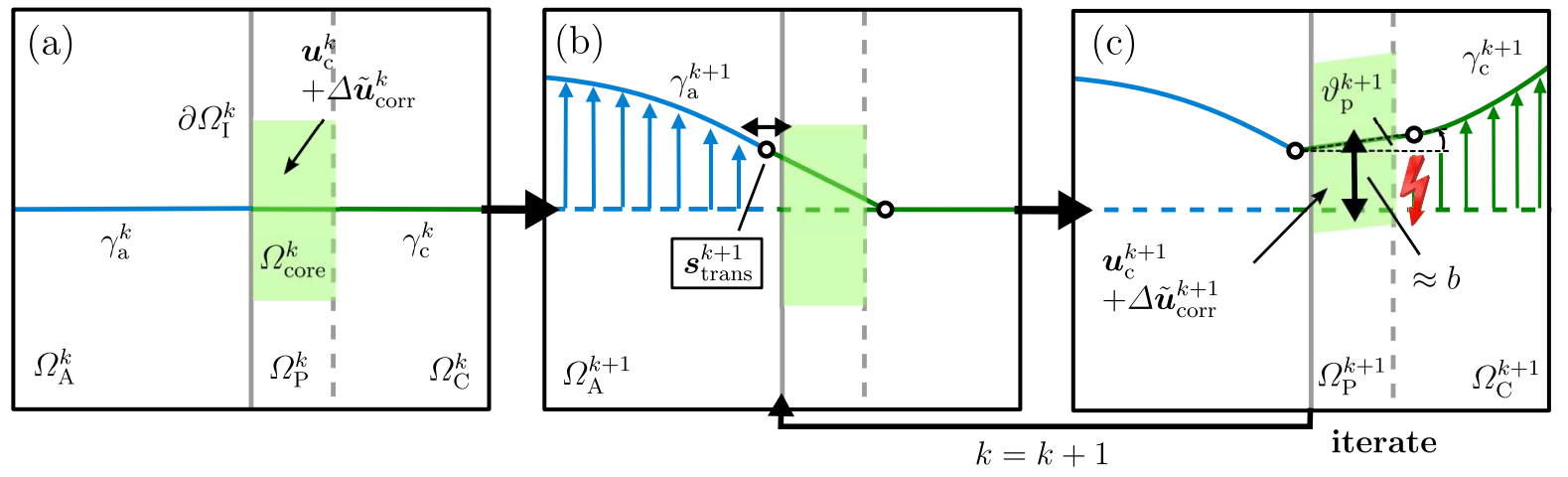

Figure 3: Schematic illustration of the multiplicative Schwarz-type algorithm for CADD-3d

Recall that the atomistic discrete dislocation, generated by the dislocation detection algorithm, is not continuous in the displacements $\boldsymbol{u}_{\mathrm{a}}$ (cf. [2, Appendix C]). Therefore the algorithm may oscillate around the equilibrium state, more precisely between two subsequent states $\left(\boldsymbol{u}_{\mathrm{a}}, \gamma_{\mathrm{hyb}}\right)^{k-1}$ and $\left(\boldsymbol{u}_{\mathrm{a}}, \gamma_{\mathrm{hyb}}\right)^{k}$. Hence, we have specified a weaker convergence criterion with respect to the subsequence

$$
\forall \text { odd } k>2 \quad\left(\boldsymbol{u}_{\mathrm{a}}^{k_{l}}\right)^{l \in \mathbb{N}}=\left(\boldsymbol{u}_{\mathrm{a}}^{1}, \boldsymbol{u}_{\mathrm{a}}^{3}, \ldots, \boldsymbol{u}_{\mathrm{a}}^{k-2}, \boldsymbol{u}_{\mathrm{a}}^{k}\right) .
$$

That is, we only compare the current state with the state two iterations previous. Convergence is then attained when

$$
\left\|\boldsymbol{u}_{\mathrm{a}}^{l}-\boldsymbol{u}_{\mathrm{a}}^{l-1}\right\|_{l^{2}\left(\Omega_{\mathrm{A}}^{0}\right)}<T O L^{\operatorname{detn}},
$$

where $T O L^{\text {detn }}$ is some pre-defined tolerance. If the criterion (4) is fulfilled it is easy to see that the algorithm is converged since the hybrid dislocation line does not get updated and therefore the pad atoms remain the same as in the previous step. The criterion (4) appeared to be considerably robust in our numerical experiments. The proposed algorithm may suggest a rather slow convergence due to the iterative procedure and the sharp interface coupling. However, note that we effectively solve the physical problem $\mathcal{P}^{\mathrm{c} / \mathrm{p}}$ in the entire domain. Therefore the discrete dislocation in the continuum can advance much further than if it would "see" a fixed boundary. The algorithm has therefore analogies with respect to overlapping domain decomposition methods. In our numerical tests we have found that our solution procedure remains reasonably fast. We will briefly comment on computational savings in Section 3.3.

There is some freedom in choosing the transmission node $\boldsymbol{s}_{\text {trans }}$ that merges $\gamma_{\mathrm{a}}$ and $\gamma_{\mathrm{c}}$, corresponding to the uncertainty in the definition of $\gamma_{\mathrm{a}}$ due to the non-unique continuum representation of $\Omega_{\mathrm{A}}$. The choice of $s_{\text {trans }}$ affects the evolution - the dislocation line may advance rather slowly in the neighborhood of the interface $\partial \Omega_{\mathrm{I}}$ if $\boldsymbol{s}_{\text {trans }}$ is too close to the artificial interface. Loosely speaking, the atomistic dislocation in Figure 3 (b) can not introduce a "discontinuity" with respect to the discrete dislocation in the continuum region. Hence, the dislocation segment in the pad may not advance by more than $\approx b$. This reveals a possible source for pre-mature convergence, in particular when the system is close to equilibrium. In the Section 3.3 we will show how different choices of $s_{\text {trans }}$ can influence the equilibrium shape of the hybrid dislocation.

The implementation of Algorithm 2 is accomplished in our in-house code libMultiscale (1sms.epfl.ch/ libmultiscale) which combines the solvers for the individual subproblems (LAMMPS and ParaDis [19, 3]).

\subsection{Validation of the approximate elasticity method}

The approximate elasticity method involves two approximations. First, it assumes that the forces on the continuum DDD nodes due to the atomistic dislocations can be accurately computed by representing the 
atomistic dislocations via the DDD method. Second, it assumes that the displacement fields on the pad atoms due to the atomistic dislocations can accurately be represented by elastic DDD fields. Use of the DDD method for both aspects implies that linear elasticity (and, usually, isotropic linear elasticity) is sufficiently accurate for these fields. Errors can thus arise, relative to a fully coupled solution, due to the inadequacy of linear, isotropic elasticity.

The DDD method also usually treats dislocations as having compact cores, whereas dislocations in fcc and hcp metals dissociate into partial dislocations separated by stacking faults. The DDD method can handle partial dislocations and stacking faults (see e.g. [20]) but this adds to the computational load since it doubles the number of segments and nodes, and greatly reduces the time increments, and hence is not usually considered in full DDD problems. In CADD-3d, the atomistic dislocation may be dissociated. The use of the core template mitigates the serious difference between the continuum line solution and atomistic solution in the core of the hybrid dislocations but the far-field interactions between dissociated atomistic dislocations and continuum line dislocations remains approximate.

The above approximate aspects are expected to create only small errors for dislocation segments that are sufficiently far from the atomistic/continuum interface. Here, we assess these errors quantitatively in terms of the spurious Peach-Koehler forces that develop as a function of the distance of an atomistic dislocation from the a/c interface. To do so, we follow Dewald and Curtin [9] and [18] and consider a straight edge dislocation residing in a semi-periodic fcc atomistic domain and approaching the atomistic/continuum interface; there are no hybrid dislocations in this problem. The problem is initialized by placing one dislocation at the center of a large box by displacing all atoms according to the continuum Volterra field as shown in Figure 4 (a) and (b). A sufficient in-plane size $(240 \AA \times 240 \AA)$ is validated ex post facto by observing that the initial dislocation motion starts at precisely the Peierls stress measured independently in a much larger atomistic cell. The box is periodic in the line direction of the dislocation, enabling use of a minimum periodic distance defined by the atomic unit cell. The initial box also includes a step consisting of two extra planes of atoms on the upper half of the box. The $\tilde{\boldsymbol{u}}$ field of the dislocation is then imposed, with the jump in the displacement across the glide plane eliminating the step to leave a smooth boundary. With the pad atoms held fixed, the atomistic system is then fully relaxed, during which the dislocation dissociates naturally into two partial dislocations separated by a stacking fault. For the large box size used here, this relaxation is independent of the $\tilde{\boldsymbol{u}}$ field. A uniform shear stress is then applied to the entire system. The dislocation commences glide at the Peierls stress $\tau_{\mathrm{P}}$. In an infinite atomistic system, the dislocation would glide continuously at the Peierls stress. In the coupled method, errors in the coupling method give rise to spurious forces $\tau_{\mathrm{SP}}$ on the dislocation; these are found to repel the dislocation from the boundary. Thus, under an applied stress $\tau_{\text {PK }}$ above the Peierls stress, the dislocation will glide until it reaches an equilibrium position (position of the center of mass of the dislocation) at distance $d$ from the interface at which the total driving force $\tau_{\mathrm{PK}}-\tau_{\mathrm{P}}-\tau_{\mathrm{SP}}=0$ (see Figure 4 ). The spurious force at $d$ is then measured directly as $\tau_{\mathrm{SP}}(d)=\tau_{\mathrm{PK}}-\tau_{\mathrm{P}}$.

(a) Reference configuration

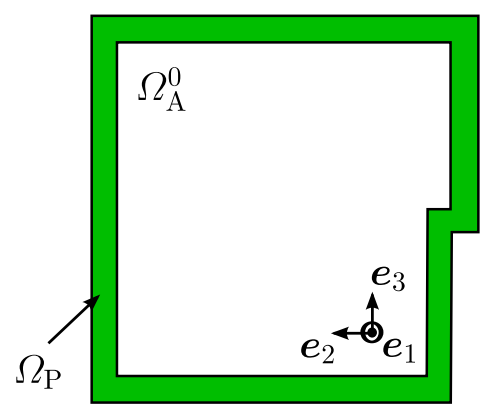

(b) Initial configuration

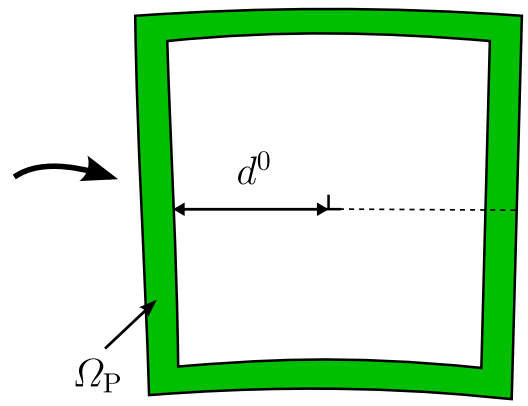

(c) Final configuration

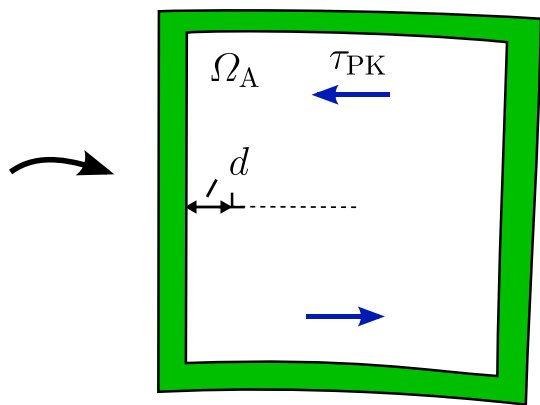

Figure 4: Schematic illustration of the numerical test to determine the spurious stress exerted on an edge dislocation near artificial interfaces. The reference configuration is given in (a). Subsequently a predictor for an edge dislocation is applied to the reference configuration in (b). An applied shear stress $\tau_{\mathrm{PK}}$ will eventually move the dislocation to the stable position in (c)

We analyze the spurious stresses corresponding to the three relevant approximate solutions for $\tilde{\boldsymbol{u}}$ that could be used in CADD-3d: 
(i) Isotropic undissociated [17]

$$
\tilde{\boldsymbol{u}}(\boldsymbol{x})=\frac{b}{2 \pi}\left(\begin{array}{c}
\frac{x_{2} x_{3}}{2(1-\nu) r^{2}}+\arctan \frac{x_{3}}{x_{2}} \\
\frac{x_{3}^{2}-x_{2}^{2}}{4(1-\nu) r^{2}}-\frac{1-2 \nu}{4(1-\nu)} \ln r^{2} \\
0
\end{array}\right), \quad r=\sqrt{x_{2}^{2}+x_{3}^{2}} .
$$

(ii) Anisotropic undissociated

$$
\tilde{\boldsymbol{u}}(\boldsymbol{x})=\tilde{\boldsymbol{u}}^{\text {aiso }}(\boldsymbol{x} ; \boldsymbol{b})=\frac{1}{2} \sum_{i=1}^{3} \ln \left(\left(x_{2}+p_{i} x_{3}\right)^{2}+\left(q_{i} x_{3}\right)^{2}\right) \boldsymbol{c}_{i}-\arctan \left(\frac{q_{i} x_{3}}{x_{2}+p_{i} x_{3}}\right) \boldsymbol{d}_{i} .
$$

We have computed the real quantities $\boldsymbol{c}_{i}, \boldsymbol{d}_{i}, p_{i}$ and $q_{i}$ for $\boldsymbol{b}=\left(\begin{array}{lll}0 & b & 0\end{array}\right)^{\top}$ using the procedure described in [12, page 444-445].

(iii) Anisotropic partial dislocations

$$
\tilde{\boldsymbol{u}}(\boldsymbol{x})=\tilde{\boldsymbol{u}}^{\text {aiso }}\left(x_{1}, x_{2}+\Delta^{\mathrm{split}}, x_{3} ; \boldsymbol{b}_{\mathrm{le}}\right)+\tilde{\boldsymbol{u}}^{\text {aiso }}\left(x_{1}, x_{2}-\Delta^{\mathrm{split}}, x_{3} ; \boldsymbol{b}_{\mathrm{tr}}\right),
$$

where $2 \Delta^{\text {split }}$ is the atomistic spacing between the leading and trailing partial dislocations having Burgers vectors $\boldsymbol{b}_{\mathrm{le}}, \boldsymbol{b}_{\mathrm{tr}}$, respectively,

$$
\boldsymbol{b}_{\mathrm{le}}=\frac{1}{2} b\left(\begin{array}{lll}
\frac{1}{\sqrt{3}} & 1 & 0
\end{array}\right)^{\top}, \quad \boldsymbol{b}_{\mathrm{tr}}=\frac{1}{2} b\left(\begin{array}{lll}
-\frac{1}{\sqrt{3}} & 1 & 0
\end{array}\right)^{\top} .
$$

To be clear, we use Algorithm 2 for each of the above approximate displacement fields. There is no discrete dislocation in the continuum domain and so only the atomistic problem is solved. At each applied stress, we relax the atomistic system, detect the new position of the dislocation (averaged of the centers of mass of the detected tetrahedra representing the full dislocation), and update the pad atom displacements according to the new positions according to the approximate displacement field $((5),(6)$ or $(7))$. We iterate until the convergence criterion (4) is satisfied with $T O L^{\text {detn }}=10^{-6} \mathrm{~b}$.

We use Aluminum as described by the EAM potential of Ercolessi and Adams [11] which is slightly anisotropic $\left(2 C_{44} /\left(C_{11}-C_{12}\right)=1.315\right)$. The partial spacing of the dissociated edge dislocation is $2 \Delta^{\text {split }}=$ $15 \AA$ and the Peierls stress is $\tau_{\mathrm{P}}=3 \mathrm{MPa}$. This problem was studied for this same material using the full CADD atomistic/continuum coupling wherein the linearly elastic continuum domain is coupled to the fully non-linear atomistic domain $[9,18]$. We compare our results to these full results as a measure of the error of using the linear elasticity solution.

Figure 5 shows the spurious stress vs. distance $d$ to the interface for the successively better approximations to $\tilde{\boldsymbol{u}}$. In all cases, including the fully non-linear solution, the spurious stresses scale as $\approx d^{-2}$. This scaling is similar to recent error estimates for isolated dislocations in a finite computational domain embedded in an effectively infinite medium for various artificial boundary conditions [10]. The magnitude of the error decreases with increasing accuracy of the approximation for $\tilde{\boldsymbol{u}}$. The isotropic compact core solution is least accurate while the anisotropic dissociated core is the most accurate when using elasticity, and the full coupled solution is overall most accurate. The magnitude of the spurious stresses shown here remain small, on the order of $10 \mathrm{MPa}$, with an error of $12 \mathrm{MPa}$ reached at distances of 40,30,18, and $7 \AA$ respectively, with increasing fidelity of the numerical method. To further support these results, we have also placed the initial dislocation at $10 \AA$ from the interface and applied Algorithm 2. The final positions of the relaxed dislocation are in agreement with the results from Figure 5 up to $\approx b$ due to the direction-dependent Peierls stress (which acts opposite to the direction of motion) and to the non-uniqueness of the dislocation detection. Overall, these results show that the typical distances over which moderate spurious coupling errors $(>5 \mathrm{MPa})$ is on the order of $30-60 \AA$ when using the various linear elasticity approximations to $\tilde{\boldsymbol{u}}$. Testing of local details of the hybrid dislocation coupling in CADD-3d must therefore, when using these approximations, ensure that the dislocations remain at these distances or further (see below). For the full CADD-3d methodology 
described in Paper I with full coupling, the spurious forces are those corresponding to the reference solution $[9,18]$, which are negligible at distances beyond $10 \AA$.

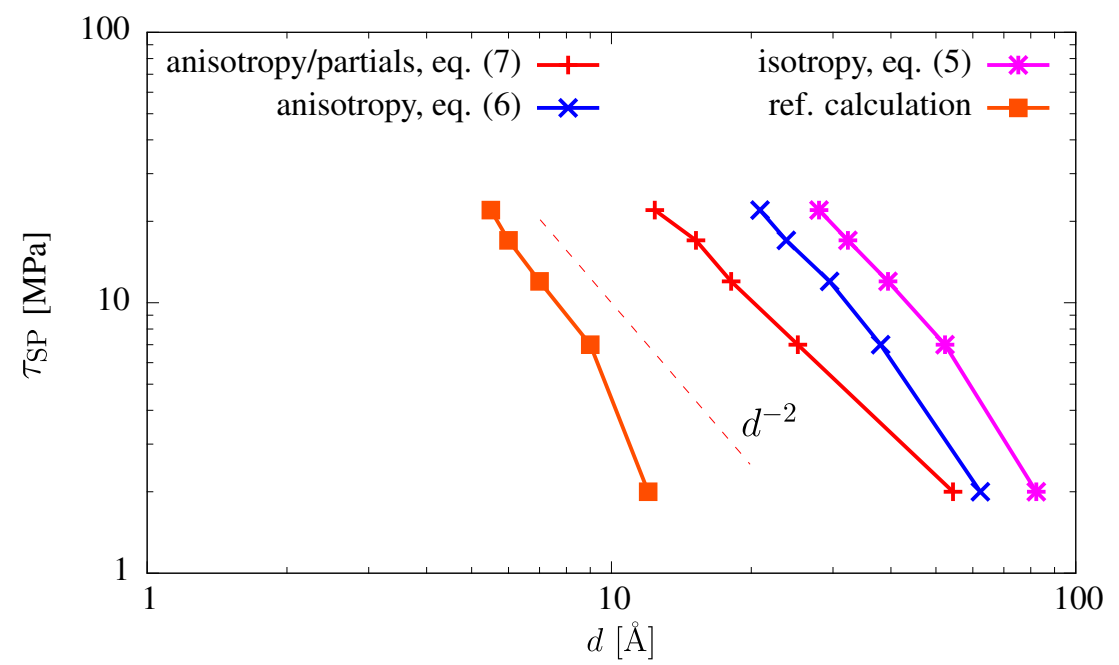

Figure 5: Spurious stress $\tau_{\mathrm{SP}}$ on a stable edge dislocation as a function of its distance to the artificial interface $d$

Finally, these tests serve as guidance for the determination of a necessary "passing" distance, or size of any overlap zone between atomistic and continuum regions, that must be developed for full operation of CADD-3d to treat dislocations moving in and out of the atomistic and continuum domains. The current approximate elasticity method (with spurious forces as shown in Figure 5) enables the use of a passing methodology. However, if the stress on the dislocations in the vicinity of the interface is rather low, the passing distance may become impracticably large as shown in Figure 5. Fortunately, this may not be an issue for many problems where dislocations mainly glide off into the bulk material (e.g. cracks under tensile loading). Another practical example is presented in the third paper of this series [7] for a Frank-Read source, subject to a constant applied shear stress. The stresses on the dislocations approaching the interface are high enough such that the passing distance can be kept to practical limits, i.e. a few Angstroms of the leading partial dislocation from the interface. For the fully coupled problem (c.f. Algorithm 1) we expect a universal passing distance in the range 5-10 $\AA$ as demonstrated by Pavia and Curtin [18] and in many other works on CADD-2d.

\section{Validation of CADD-3d for hybrid dislocations}

\subsection{Reference problem}

As a test problem to assess the accuracy of the CADD-3d treatment of hybrid dislocations, we study the quasistatic bow-out of an initially straight planar periodic array of edge dislocations in an infinite box under an applied resolved shear stress $\tau_{\mathrm{PK}}$. A schematic of the problem is shown in Figure 6 (a). This problem can be studied accurately in a full atomistic simulation in a suitable large but finite size box and in a full discrete dislocation dynamics simulation (see below), which enables (i) careful comparison of the predictions of the CADD-3d method to the fully atomistic results and (ii) calibration of DDD to atomistics (see Figure $6(\mathrm{~b})$ and $(\mathrm{c}))$.

We choose a single periodic spacing of the pinning points of $l_{1} \approx 200 \AA{ }^{3}$ which is then the periodic length of the simulation cell along $x_{1}$. We then use a finite box size, $l_{2}$ along the glide direction and $l_{3}$ normal to the slip plane, with periodicity in $x_{2}$ and traction boundary conditions on the $x_{3}$ surfaces. This size is sufficient to ensure that image effects, due to the traction-free $x_{3}$ surfaces, of the non-straight bowed-out dislocations are minimal and can be neglected relative to the applied loads. Following [25] for exactly this problem, we use $l_{2} \approx l_{3} \approx 400 \AA$. Pinning of the dislocation follows $[25,26]$ and is described in more detail below.

\footnotetext{
${ }^{3}$ Note that the real box dimensions can vary slightly from the given values according to the periodic interatomic spacing
} 
(a)

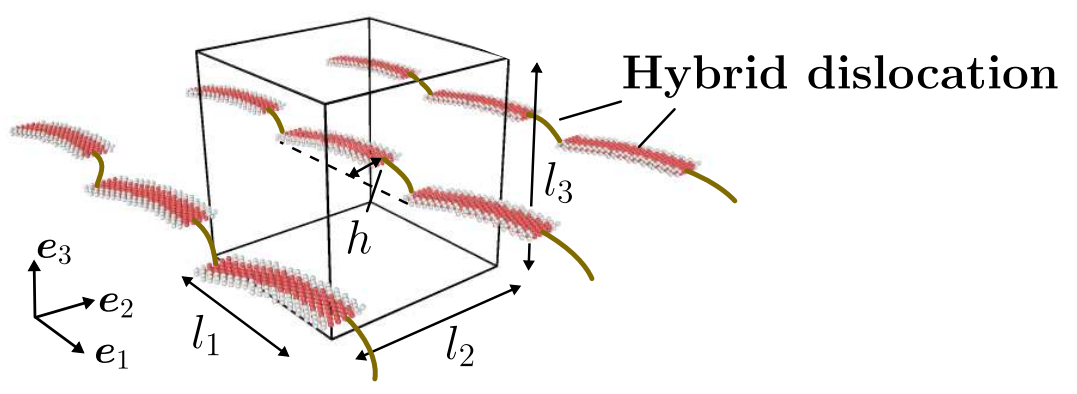

(b)

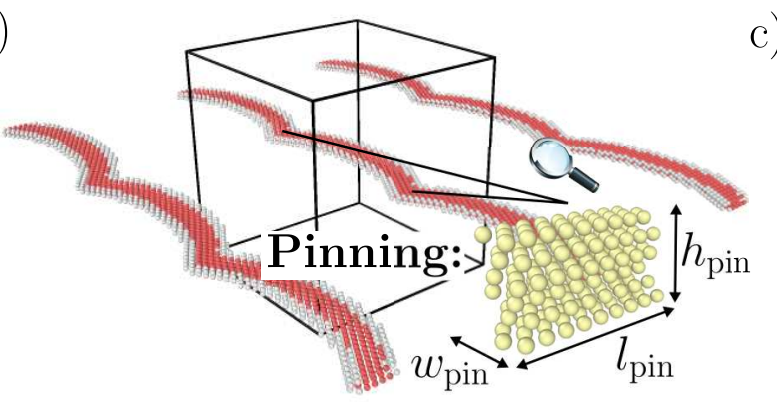

c)

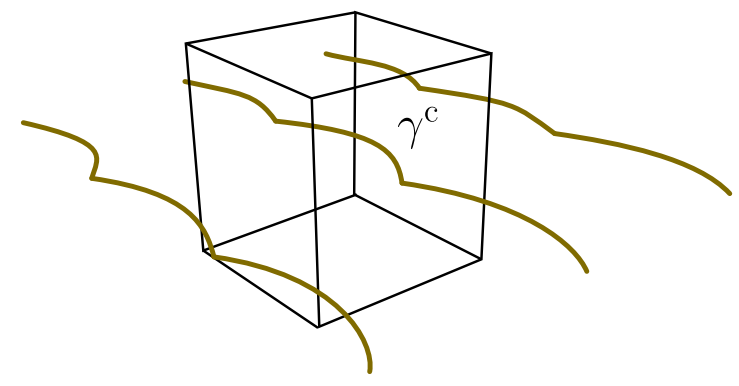

Figure 6: Schematic illustration of the bow-out of a nominal straight dislocation for (a) the coupled CADD-3d problem, (b) the fully atomistic model (only the atoms in the core region are visualized), and (c) the continuum DDD model

In accordance with our validation study in the last section, we use Aluminum modeled by an Embedded Atom Model (EAM) Ercolessi and Adams [11]. Material parameters needed for the isotropic continuum DDD simulation are the lattice constant $a_{0}=4.032 \AA$, Burgers vector $b=2.851 \AA$, approximate isotropic Poisson ratio $\nu=0.35$ and shear modulus $\quad \mu=30.8 \mathrm{GPa}$. For this quasistatic problem, results are independent of the dislocation mobility, although a value for the mobility is used for incrementing the DDD solution toward equilibrium. Also required is a calibrated dislocation core energy to supplement the non-singular dislocation field solution in the DDD code ParaDis; this is discussed below.

Before describing the full test problem in more detail, it is convenient to present the two corresponding reference problems, the fully atomistic and fully DDD models of the same test problem geometry. The fully atomistic solution provides the base for assessment of the CADD-3d algorithms. The fully DDD solution is used to calibrate the DDD core energy parameter to the fully atomistic solution.

\subsection{Atomistic and discrete dislocation dynamics reference problems}

Schematic illustrations of the two reference problems are shown in Figure 6 (b) and (c). The fully atomistic box dimensions $l_{1}, l_{2}$ and $l_{3}$ are those given above, with $\approx 2$ million atoms and therefore $\approx 6$ million degrees of freedom. The introduction of the periodic array of straight dislocations in the initial structure is accomplished using the known elastic displacement fields of the so-called periodic array of dislocations (PAD, see Appendix A) given by

$$
\begin{aligned}
& \tilde{u}_{1}^{\mathrm{PAD}}(\boldsymbol{x})=0, \\
& \tilde{u}_{2}^{\mathrm{PAD}}(\boldsymbol{x})=-\frac{b}{4 \pi(1-\nu)} \frac{C x_{3} \sin \left(2 C x_{2}\right)}{\left(\cos \left(2 C x_{2}\right)-\cosh \left(2 C x_{3}\right)\right)}-\frac{b}{2 \pi} \arctan \left(\operatorname{coth}\left(C x_{3}\right) \tan \left(C x_{2}\right)\right), \\
& \tilde{u}_{3}^{\mathrm{PAD}}(\boldsymbol{x})=-\frac{b}{4 \pi(1-\nu)} \frac{C x_{3} \sinh \left(2 C x_{3}\right)}{\left(\cos \left(2 C x_{2}\right)-\cosh \left(2 C x_{3}\right)\right)}-\frac{b(1-2 \nu)}{8 \pi(1-\nu)} \ln \left(\left|\cos \left(2 C x_{2}\right)-\cosh \left(2 C x_{3}\right)\right|\right),
\end{aligned}
$$

where $C=\pi / l_{2}$. Note that (9) is not truly periodic since it contains the slip step but, as in the previous section, we choose a reference configuration that includes the same slip step so that the deformed configuration has the required in-plane periodicity.

The atom positions are then relaxed to equilibrium subject to the periodic boundary conditions in $x_{1}$ - and $x_{2}$-directions and free surfaces on the top and bottom $x_{3}$ boundaries. The Hessian-free Newton-Raphson algorithm in LAMMPS is used, with the convergence criterion $\left\|\boldsymbol{f}_{\mathrm{a}}\right\|_{l^{2}\left(\Omega_{\mathrm{A}}\right)}<10^{-4} \mathrm{eV} / \AA$. 
After the initial relaxation, we establish the "pinning points" for subsequent bow-out (see Figure 6 (b)) as follows. Atoms in a small rectangle (dimensions: $l_{\text {pin }}=24 \AA, w_{\text {pin }}=12 \AA, h_{\text {pin }}=6 \AA$ ) centered on the core of the dislocation and at one end of the periodic box along $x_{1}$ are identified. These atoms are then held fixed (zero subsequent displacement) during subsequent loading of the simulation cell. Forces are then applied to the atoms on the top along $x_{3}$ corresponding to a desired applied shear stress $\tau_{\mathrm{PK}}$ as $\boldsymbol{f}^{\text {ext }}=\frac{A}{N} \tau_{\mathrm{PK}} \boldsymbol{e}_{2}$ where $A=l_{1} l_{2}$ is the area of the top surface and $N$ the number of atoms in the surface layer, with forces of opposite sign applied on the bottom surface atoms. The entire system is then again allowed to relax to equilibrium, during which the dislocation core bows out between the periodic pinning points to reach an equilibrium configuration characterized by the bow-out height $h$ at the center of the box.

In the corresponding full DDD continuum problem, the pinning points are defined by fixed segments or length $w_{\text {pin }} / 2$ on each end of the dislocation line along $x_{1}$. The initial dislocation line is discretized into 16 piecewise linear segments of lengths between $5 b-8 b$. The DDD methodology in ParaDis is employed, which uses a nonsingular theory [6] with parameter $a$ to regularize the singular core. An additional core energy per unit length is introduced with a dependence on the character angle according to linear elastic theory as

$$
W^{\text {core }}(\vartheta)=E^{\text {core }}\left(\frac{\sin ^{2} \vartheta}{1-\nu}+\cos ^{2} \vartheta\right) b^{2},
$$

where $E^{\text {core }}=E^{\text {core }}(a)$ is an additional scalar parameter. Since the non-singular theory includes some core energy through the regularization parameter $a$, the parameter $E^{\text {core }}(a)$ has an implicit dependence on $a$ if the total DDD core energy is intended to agree with the true atomistic core energy. Usually $E^{\text {core }}$ is calibrated with respect to a representative atomistic configuration, e.g. straight dislocations in infinite domains. For the bow-out problem, Szajewski et al. [26] were calibrating $E^{\text {core }}$ for a given amount of bowout and showed good agreement between fully atomistic and fully DDD solutions in ParaDis by varying the periodic length $l_{1}$ between the pinning points. Here, we adopt their choice of parameters, i.e.

$$
a=7.714 \AA, \quad E^{\text {core }}(a)=5 \mathrm{GPa} .
$$

throughout this paper.

Solution of the DDD bow-out problem within ParaDis is achieved by computing the velocities for nodes $s \in \gamma_{\mathrm{c}}$ using the overdamped mobility law

$$
\boldsymbol{v}=\boldsymbol{M}\left(\boldsymbol{f}^{\mathrm{pk}}+\boldsymbol{f}^{\mathrm{core}}\right) .
$$

where $\boldsymbol{M}$ is the mobility tensor. Here, we restrict motion to gliding on the defined glide plane and seek quasistatic solutions. The numerical solution of (12) is reduced, using a forward-Euler integration scheme with time step $\Delta t$, to a steepest descent method

$$
\boldsymbol{v}^{k} \approx \frac{\boldsymbol{s}^{k+1}-\boldsymbol{s}^{k}}{\Delta t} \quad \Rightarrow \quad \boldsymbol{s}^{k+1}=\boldsymbol{s}^{k}+\Delta t \boldsymbol{M}\left(\boldsymbol{f}^{\mathrm{pk}, k}+\boldsymbol{f}^{\mathrm{core}, k}\right),
$$

where $\Delta t \boldsymbol{M}$ acts as a constrained line search. The steepest descent method is known to converge rather slowly but here the number of nodes is small and the computational cost of solving the DDD problem is negligible as compared to the cost of solving the atomistic problem. Convergence is achieved when the total force on the dislocation line is

$$
\left\|\boldsymbol{f}^{\mathrm{pk}}+\boldsymbol{f}^{\mathrm{core}}\right\|_{L^{2}\left(\gamma_{\mathrm{c}}\right)}<10^{-3} \mathrm{~N} .
$$

Figure 7 shows the fully-atomistic and fully DDD configurations obtained at applied shear stresses of 50, 100 and $150 \mathrm{MPa}$. The good agreement confirms that the choice of the core energy parameter in ParaDis is sufficiently accurate. At $150 \mathrm{MPa}$, there is a slight deviation in the maximum bow-out between the two models $(\approx 34 \AA$ for DDD vs. $\approx 30 \AA$ for atomistics), which most likely arises because the core parameters 
(11) were calibrated using different box sizes than those used here ${ }^{4}$ but given the simplicity of the approach which only requires the calibration of one single parameter, the results seem remarkable. As a matter of fact, a perfect match between both models - which one could obtain by calibrating $E^{\text {core }}$ to the configurations shown in Figure 7 - is not essential to validate crucial features of the coupled problem as done in the following section. In practice we always expect small deviations between atomistic and DDD models.
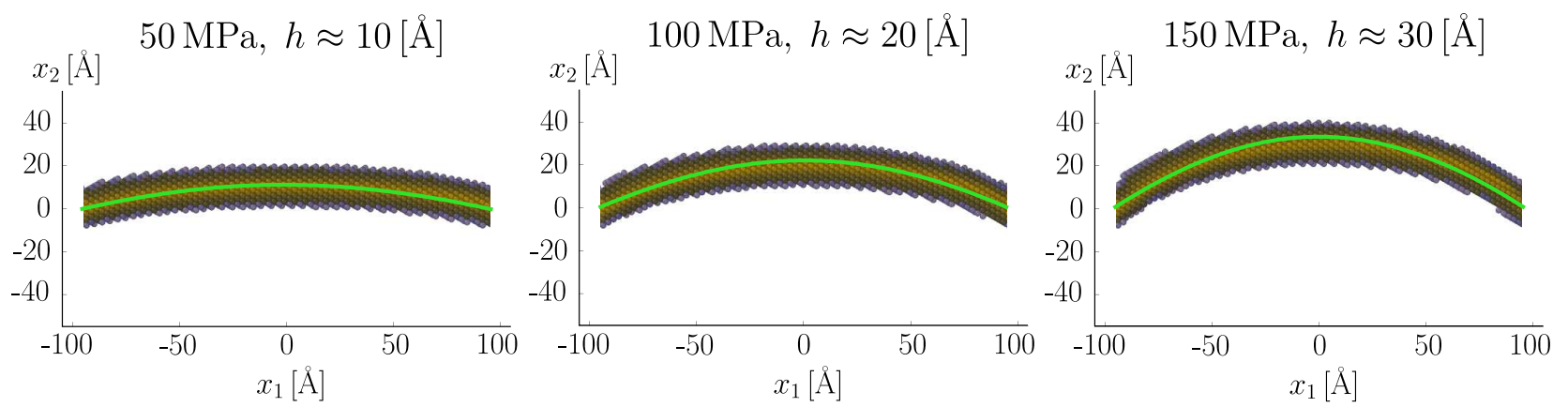

Figure 7: Comparison between fully atomistic calculations and the continuum model (solid line) for different applied shear stresses

\section{3. $C A D D-3 d$ problem}

For the CADD-3d study of the bow-out problem, we divide the entire domain into atomistic and continuum regions along the $x_{1}$-direction (see Figure $6(\mathrm{a})$ ). We denote $w_{\mathrm{a}}$ as the width of the atomistic domain. A schematic top view of the coupled problem is shown in Figure 8 (a). The width of the pad domain is conveniently set to the width of the pinning points $w_{\text {pin }}$ which is slightly greater than two times the cut of radius $r_{\mathrm{c}}=5.56 \AA$ of the interatomic potential. The initial displacements of the atomistic domain are taken as the relaxed configuration of the periodic array of straight dislocations from the previous subsection. The initial hybrid dislocation is a straight line along the origin.

We apply a homogeneous shear stress at infinity, which generates displacements of the pad atoms given by

$$
\hat{\boldsymbol{u}}\left(\boldsymbol{r}_{\mathrm{a}}\right)=\frac{\tau_{\mathrm{PK}}}{2 \mu} r_{\mathrm{a}, 3} \boldsymbol{e}_{2} \quad \forall \boldsymbol{r}_{\mathrm{a}} \in \Omega_{\mathrm{P}}
$$

As the dislocation bows out, the additional displacements of the pad atoms are computed as the sum of the elastic displacements for the periodic array of straight edge dislocations $\tilde{\boldsymbol{u}}^{\mathrm{PAD}}$ (9) plus a correction $\Delta \tilde{\boldsymbol{u}}^{(i, j)}$ due to curved segments for the primary and periodic images $(i, j)$. The correction is computed using the Barnett formalism $[4,5]$ as described in Appendix B. Including the core template correction, the displacements of the pad atoms are thus given by

$$
\boldsymbol{u}_{\mathrm{a}}\left(\boldsymbol{r}_{\mathrm{a}}\right)=\tilde{\boldsymbol{u}}^{\mathrm{PAD}}\left(\boldsymbol{r}_{\mathrm{a}}\right)+\sum_{j=-N_{2}}^{N_{2}} \sum_{i=-N_{1}}^{N_{1}} \Delta \tilde{\boldsymbol{u}}^{(i, j)}\left(\boldsymbol{r}_{\mathrm{a}}\right)+\Delta \tilde{\boldsymbol{u}}_{\mathrm{corr}}\left(\boldsymbol{r}_{\mathrm{a}}\right)+\hat{\boldsymbol{u}}\left(\boldsymbol{r}_{\mathrm{a}}\right) \quad \forall \boldsymbol{r}_{\mathrm{a}} \in \Omega_{\mathrm{P}},
$$

where $N_{1}, N_{2}$ are the number of periodic images considered in the $x_{1}$ - and $x_{2}$-direction, respectively. For the application of the core template [2], we use a core region $\Omega_{\text {core }}$ with $R^{\text {core }}=16 \AA$ which covers the stacking fault of the chosen interatomic potential. The core region comprises a blending region of width $\approx 4 \AA$ to guarantee a smooth transition of $\Delta \tilde{\boldsymbol{u}}_{\text {corr }}$ to zero at $\partial \Omega_{\text {core. }}$. We have run tests with larger core templates with no qualitative change in the results. Throughout the simulations, the displacements of atoms in the pinning regions are held fixed.

After each relaxation of the atomistic system, we re-detect the discrete dislocation $\gamma_{\mathrm{a}}$. Following our approximate approach (see Section 2.2), the full DDD line $\gamma_{\mathrm{a}}+\gamma_{\mathrm{c}}$ is used to compute the forces on $\gamma_{\mathrm{c}}$ and the pad displacements. The DDD line $\gamma_{\mathrm{c}}$ is then evolved according to the PK forces on the nodes within

\footnotetext{
${ }^{4}$ This is expected and was also observed in the work by Szajewski et al. [26] (c.f. Figure 6 (a) and (b) in[26])
} 
(a)
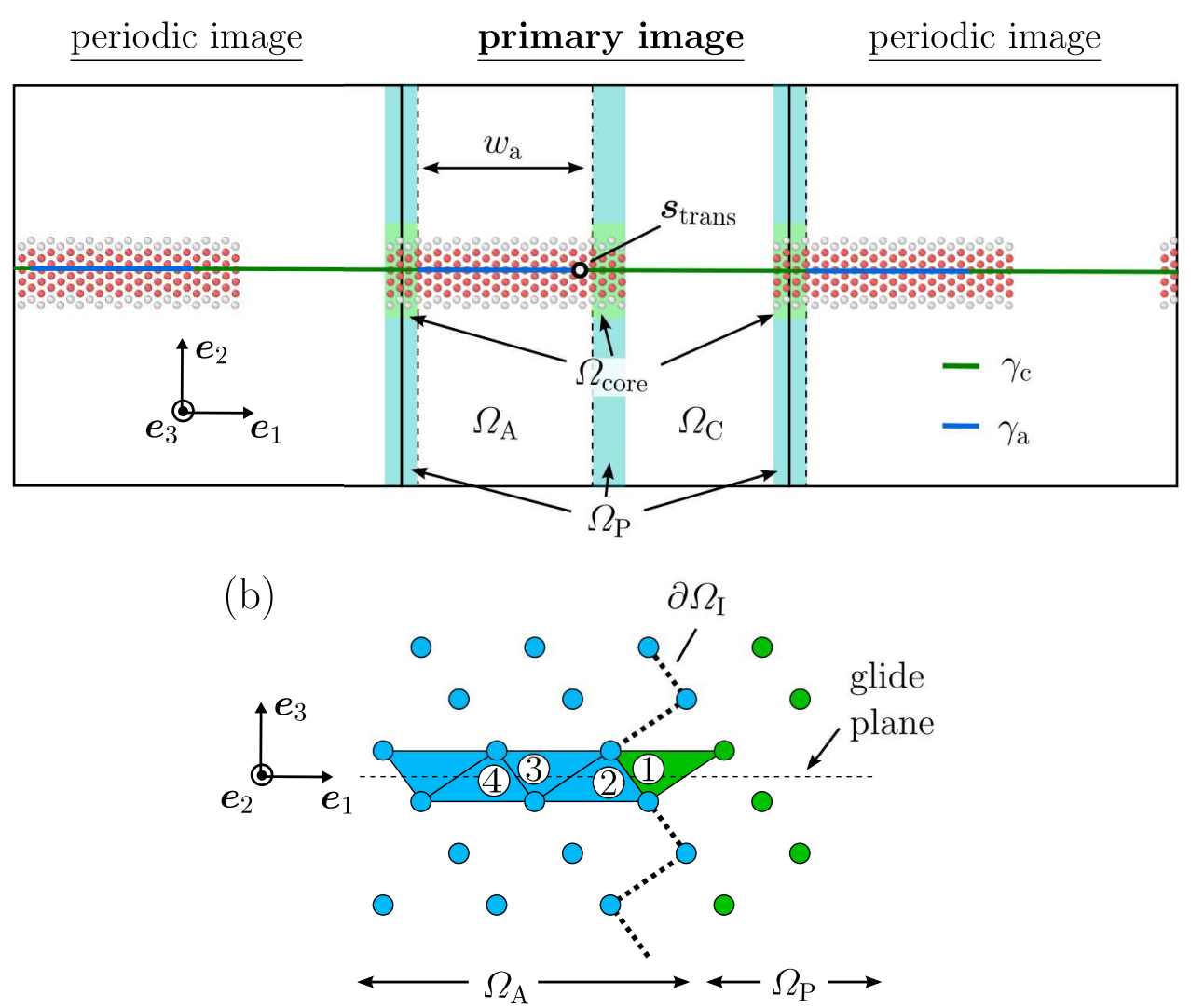

Figure 8: Schematic top view of the domain decomposition for the PAD geometry (a). A side view along the dislocation line direction is shown in (b) to illustrate where the detection algorithm generates nodal positions of $\gamma_{\mathrm{a}}$ (the interface node is located in region 1 , its neighboring node in region 2 etc.)

ParaDis. The new DDD line is then used to update the pad displacement field, and the atomistic system is then relaxed again.

As indicated in Section 2.2 the choice of the transmission node $\boldsymbol{s}_{\text {trans }}$ is not unique and may influence the final converged solution. The algorithm in Paper 1 states that $\boldsymbol{s}_{\text {trans }}$ should reside in the atomistic domain, and here we demonstrate explicitly the effect of $s_{\text {trans }}$ on the final solution. For the selection of the transmission node we employ a simple scheme which does not require additional efforts with regard to the implementation. Recall that the dislocation detection algorithm identifies successive tetrahedral units in the atomistic domain, schematically depicted by the filled triangles in Figure 8 (b). These units are then used to discretize the dislocation into nodes and segments. The transmission node is then the first node in the atomistic domain, which could be in the first tetrahedron, the second, the third, etc. Assume for a moment that $\boldsymbol{s}_{\text {trans }}$ is in the first tetrahedron (i.e. region 1 in Figure 8 (b)). As a consequence $\gamma_{\mathrm{a}}$ would only advance in very small increments in the vicinity of the interface. As a result, the algorithm may suffer from premature convergence leaving artificial kinks at the interface. Therefore it might be favorable to ignore the interface node and set $\boldsymbol{s}_{\text {trans }}$ to be equal to the detected node in region 2, 3 or even farther in the atomistic domain.

We apply the coupled problem to study the bow-out process at an applied shear stress of $150 \mathrm{MPa}$. At this stress level, the reference atomistic and DDD dislocation lines do not match perfectly but are sufficiently close to enable assessment of the coupled problem. Convergence is attained when the criterion (4) is satisfied with $T O L^{\text {detn }}=10^{-2} b$. For all numerical simulations here, convergence is reached after 15-25 global iterations.

In order to judge the accuracy of the coupled problem, we compare the bow-out of the hybrid dislocation with the atomistic reference calculation. More precisely, we measure the difference in the displacements $\Delta s_{\mathrm{hyb}, 2}$ and $\Delta s_{\mathrm{a}, 2}^{\mathrm{ref}}$ along the glide direction ( $x_{2}$-direction). In practice we are only interested in the error in $\Omega_{\mathrm{A}}$. Defining $\mathcal{A}(w)=\left(-l_{1} / 2+w_{\mathrm{pin}},-l_{1} / 2+w_{\mathrm{pin}}+w\right)$ as an interval along the $x_{1}$-direction which 
encompasses a length $w \leq w_{\mathrm{a}}$, we then define the relative error with respect to $w$ as

$$
e_{\mathrm{rel}}(w)=\frac{\left\|\Delta s_{\mathrm{hyb}, 2}-\Delta s_{\mathrm{a}, 2}^{\mathrm{ref}}\right\|_{L^{2}(\mathcal{A}(w))}}{\left\|\Delta s_{\mathrm{a}, 2}^{\mathrm{ref}}\right\|_{L^{2}(\mathcal{A}(w))}} .
$$

We have analyzed the coupled problem for two different domain decompositions, $w_{\mathrm{a}} \approx l_{1} / 4$ and $w_{\mathrm{a}} \approx l_{1} / 2$, and various choices of the transmission node position. The relative errors are presented in Table 1.

(a)



(c)

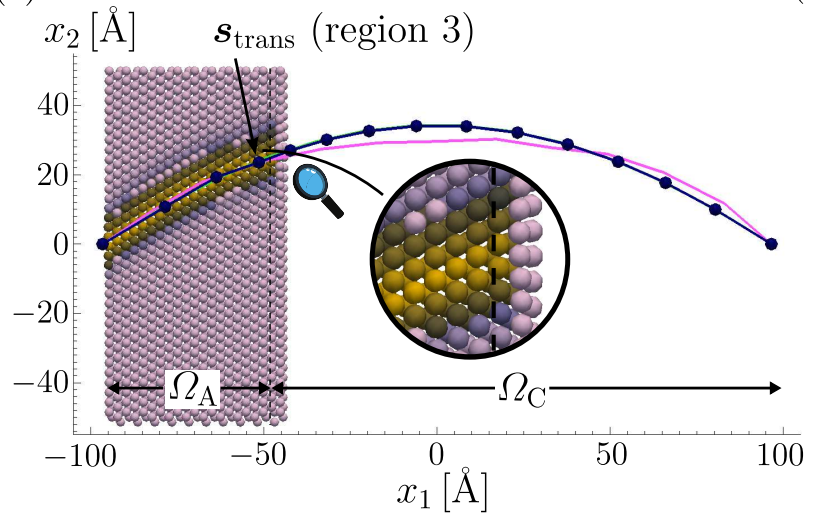

(b)

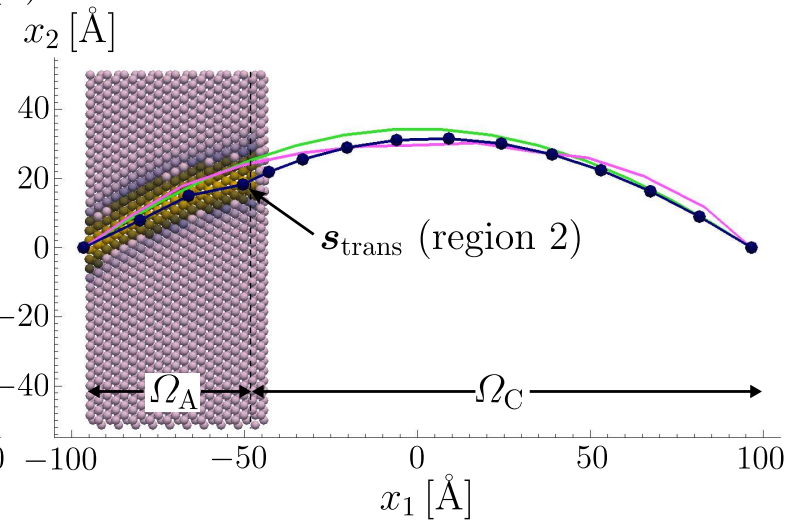

(d)

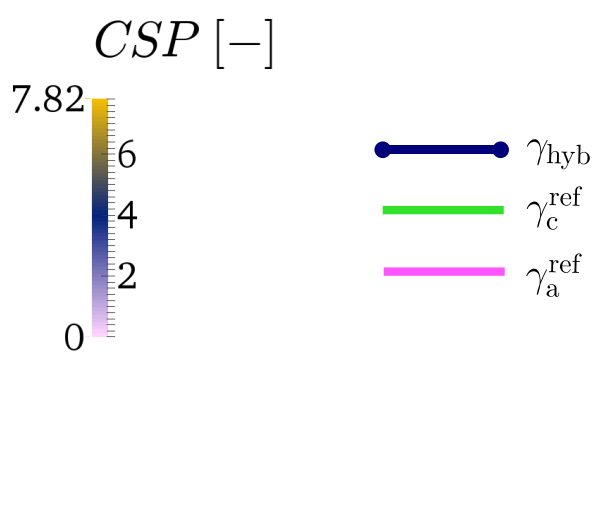

Figure 9: Solution of the coupled problem for $w_{\mathrm{a}}=l_{1} / 4$ for different choices of the transmission node $\boldsymbol{s}_{\text {trans }}$ (a)-(c). Real and pad atoms are highlighted with respect to the centrosymmetry parameter [13, CSP] according to (d)

We first choose the width of the atomistic domain to be $w_{\mathrm{a}} \approx l_{1} / 4$ of the width of the domain. This problem represents a crucial test case for the core template approximation since the character angle must evolve and eventually reach the equilibrium value $0^{\circ}<\vartheta_{\mathrm{p}}<90^{\circ}$ near the atom/continuum interface. We discretized $\gamma_{c}$ into eleven segments (including the one crossing the pad region) such that the segment length is approximately the same as for the reference DDD problem. We have also chosen a coarsening of the discrete atomistic dislocation to match this segment length approximately such that the total number of segments remains between 14-16 during one simulation. Tests were then performed using different locations of the transmission node $s_{\text {trans }}$. The final equilibrium configurations are shown in Figure 9 (a)-(c). The choice of the transmission node has a clear influence on the convergence. As stated in Paper I, when the transmission node is at or near the atom/continuum interface, namely in region 1 or 2 (c.f. Figure 8 (b)), the algorithm converges pre-maturely to leave a small kink at the a/c interface. When the transmission node is further from the a/c interface (region 3), the converged solution is in excellent agreement with the fully-atomistic solution. Specifically, the hybrid dislocation line coincides nearly perfectly with the atomistic/continuum descriptions in $\Omega_{\mathrm{A}}$ and with the DDD description in $\Omega_{\mathrm{C}}$, as shown quantitatively in Table 1 by the small error, which is on the order of the difference between fully atomistic and fully DDD problems. When the transmission node lies deeper into the atomistic domain (region 4), the results do not change notably. We conclude, as stated in Paper I, that it is necessary to choose the transmission node to lie a few atomic layers inside the atom/continuum interface to obtain accurate results. 


\begin{tabular}{c|c|c|c}
\hline width $w_{\mathrm{a}}$ of $\Omega_{\mathrm{A}}$ & region of $\boldsymbol{s}_{\text {trans }}$ & $w$ & $e_{\text {rel }}(w)[\%]$ \\
\hline \hline$l_{1} / 4$ & 1 & $l_{1} / 4$ & 26.9 \\
$l_{1} / 4$ & 2 & $l_{1} / 4$ & 21.4 \\
$l_{1} / 4$ & 3 & $l_{1} / 4$ & 5.2 \\
$l_{1} / 2$ & 3 & $l_{1} / 2$ & 5.8 \\
$l_{1} / 2$ & 3 & $l_{1} / 4$ & 4.1 \\
full DDD & $\nearrow$ & $l_{1} / 4$ & 5.7 \\
full DDD & $\nearrow$ & $l_{1} / 2$ & 9.9 \\
\hline
\end{tabular}

Table 1: Error in the bow-out between the hybrid dislocation and the detected atomistic dislocation from the reference calculation. The third column specifies the width $w$ of the domain over which the error is measured

More broadly, the result in Figure 9 (c) demonstrates the high fidelity of the proposed CADD-3d treatment of hybrid dislocations. A close inspection of the atomic displacements near the atom/continuum interface shows a very slight shift in the visualized atomic core structure, but the overall level of agreement is excellent. The atomistic dislocation away from the interface is experiencing no spurious stresses that cause a measurable disturbance, so the atomistic system does not know that the dislocation is represented by DDD in another large portion of the domain. Such atomistic fidelity is precisely the goal of CADD-3d.

To further validate CADD-3d, we examine the same problem but with an atomistic domain that is approximately the same size as the continuum domain. This captures the region of the bow-out where the atomistic and DDD reference problems show the largest differences in equilibrium positions. Seven segments are used to discretize the continuum dislocation line in $\Omega_{\mathrm{C}}$ and we choose the transmission node to be in region 3. Otherwise the problem remains the same as above. The final configuration at $150 \mathrm{MPa}$ is shown in Figure 10. The hybrid dislocation line now resides between the solution for the individual problems, with an error (c.f. Table 1) of approximately half of the error of the full DDD reference problem in half of the total domain. Moreover, our analysis shows that, considering only the error in $\mathcal{A}\left(l_{1} / 4\right)$, improved results are obtained in comparison with the coupled problem where the width of the atomistic domain was $l_{1} / 4$.

To demonstrate the benefits of the atomistic core template approximation, we have also performed simulations using solely the Volterra solution in the entire pad region. Results at $150 \mathrm{MPa}$ are shown in Figure 11 for two different positions of the transmission node. For the transmission node in region 3 , there is an artificial pinning of the dislocation leading to a kink that should not exist. The atoms in region 3 experience the constraint of the incorrect core template and cannot adjust suitably. Away from the kink, however, the solution is in reasonable agreement with the previous results. The use of the Volterra core retains knowledge of the Burgers vector and correct slip displacements, and so the differences between the Volterra core and the full dissociated core are limited to short-range fields, and thus cause short-range disturbances. However,

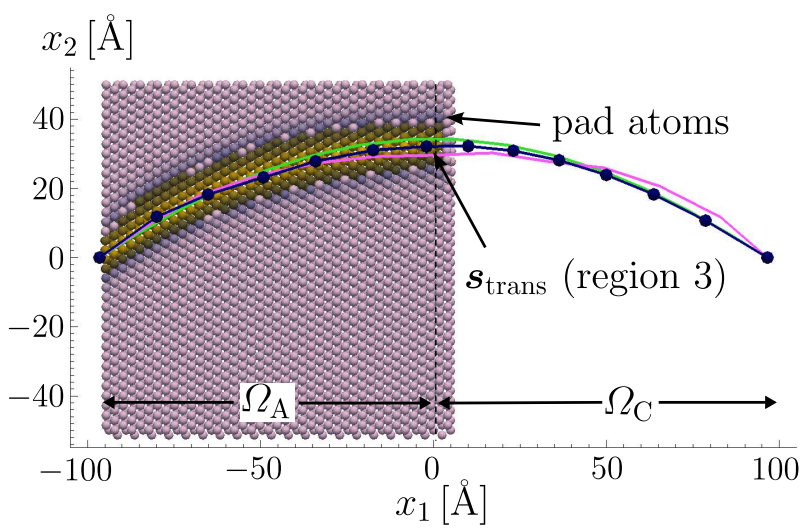

Figure 10: Solution of the coupled problem for $w_{\mathrm{a}}=l_{1} / 2$. Real and pad atoms are highlighted with respect to the centrosymmetry parameter $[13, \mathrm{CSP}]$ according to Figure 9 (d) 
those disturbances do extend into the atomistic region and thus generate unwanted spurious stress fields that may drive unphysical atomistic behavior even though the atomic displacement differences are localized. In the present approximate model that uses DDD fields to inform the pad atoms, the atomic disturbances caused by the Volterra solution are not fed back into the pad - there is no full coupling - and hence the disturbances are likely underestimated when using the current approximate model. Interestingly, if the transmission node is moved slightly further from the interface (region 4), then the final hybrid dislocation line is largely unaffected by the use of the Volterra core. In region 4, the atoms are able to correct slightly better for the Volterra field error, and the segment connecting the transmission node to the first DDD node spans across the Volterra solution, smoothening out the DDD description. While the Volterra solution is attractive for simplicity, the core template approximation yields a smoother transition between the two descriptions of the dislocation and a far better description of the atomistic displacements inside the atomistic region near the atom/continuum interface. The smoother transition can be visualized by comparing the close-up views in Figure 11 (b) and Figure 9 (c); the dislocation core becomes significantly more compact from $\approx 3 b$ to the interface.

(a)

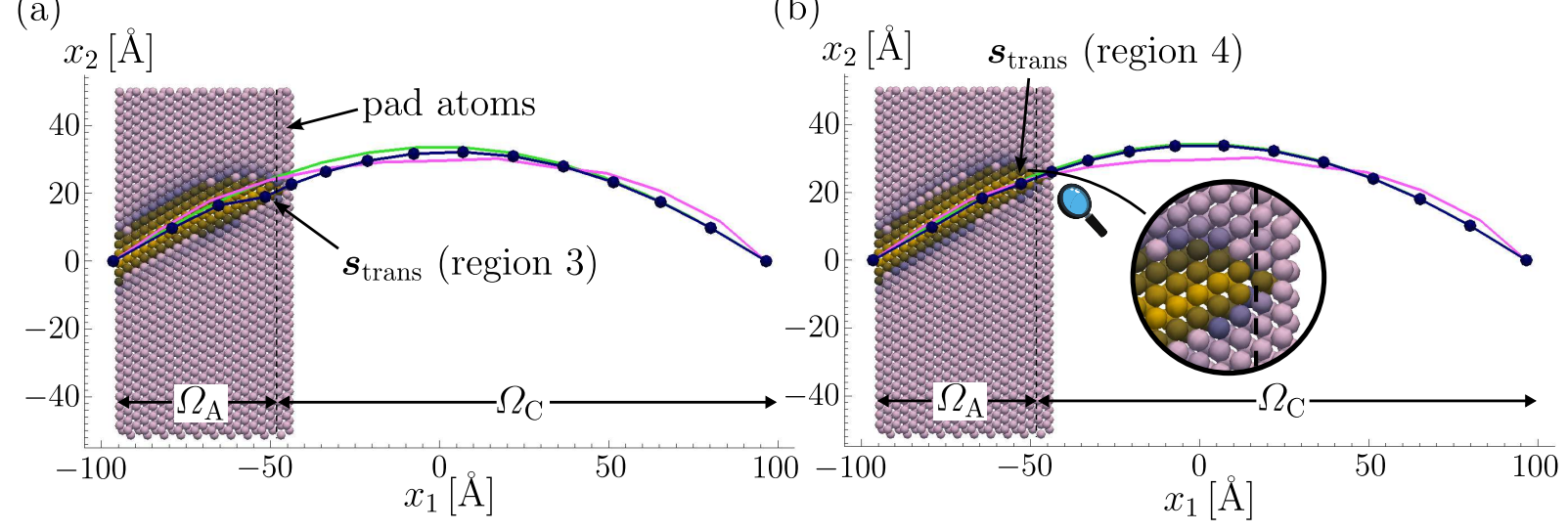

Figure 11: Solution of the coupled problem for $w_{\mathrm{a}}=l_{1} / 4$ using the classical Volterra solution in the core region. Real and pad atoms are highlighted with respect to the centrosymmetry parameter [13, CSP] according to Figure 9 (d)

We do not present a detailed comparison of computation efficiency of the coupled problem at this stage of the CADD-3d development. The test problems here are very small in size, especially the DDD regions, and so CADD-3d is not expected to be notably faster than a full atomistic solution. Nonetheless, the computational time for the results in Figure $10\left(w_{\mathrm{a}}=l_{1} / 2\right)$ were comparable to those for the fully atomistic problem and the computational time for the results in Figure $9\left(w_{\mathrm{a}}=l_{1} / 4\right)$ were $\approx 3.5$ less than the fully atomistic problem, thus approaching perfect scaling with the atomistic size. Thus, even these preliminary tests indicate the high possible efficiency of CADD-3d for problems when the entire domain is much much larger than the atomistic domain alone.

\section{Summary}

We have presented a validation of the three-dimensional Coupled Atomistic and Discrete Dislocation (CADD3d) method introduced in [2]. We have specifically shown that CADD-3d can handle hybrid dislocations that span the atomistic and continuum domains with high fidelity, approaching the exact fully atomistic solution. This has been accomplished in a simple yet non-trivial quasi-static formulation enabling us to focus on equilibrium configurations. We eliminate the need to execute $3 \mathrm{~d}$ finite-element solutions for the corrective discrete dislocation fields through an approximate method that uses a fully-DDD representation of the dislocation network at any instant to compute the boundary conditions on the atomistic domain. We have quantitatively assessed the accuracy of this approximation. Even with this approximation, the atomistic domain still evolves according to atomistic forces, and so the main new features of the continuum/atomistic coupling are preserved here.

The quasi-static CADD-3d method has been tested by studying the problem of the bow-out of a dislocation that is pinned periodically along its length. By comparing CADD-3d predictions to a fully atomistic solution 
of the same problem, we demonstrated the accuracy of the method in all important aspects. We have further shown that two algorithmic details (the use of the core template to accurately represent the true atomistic core structure of the dislocation and the choice of the transmission node connecting the continuum and atomistic portions of a hybrid dislocation) are essential for achieving high accuracy. The present study does not fully show the errors associated with use of the Volterra core instead of the accurate core template because a full true coupled boundary value problem is not studied; this will be studied when such full coupling is implemented. With the basic validation accomplished here for a simple quasi-static problem, Paper III in this series will present many further validations and applications of the current CADD-3d formulation for dynamic problems.

The present methodology, including the elasticity approximation for obtaining atomistic boundary conditions, can be used to enrich the fidelity of existing DDD studies. That is, during the evolution of a full DDD simulation, a fully-atomistic domain can be inserted into any region of the DDD simulation in which one wishes to interrogate the atomistic details specifically. The DDD network is used to define the boundary conditions of the atomistic domain, and then the CADD-3d algorithm is used to evolve the coupled problem and resolve atomistic behavior in the selected region. The problem can revert to a full DDD simulation automatically because the current CADD-3d method is always following the entire $3 \mathrm{~d}$ network. The current CADD-3d is therefore the computational nanoscope that enables on-the-fly atomistic study of any domain of interest as a DDD system evolves in time.

There remain some current operational limits to the coupling of atomistics to discrete dislocation dynamics with full atomistic fidelity due to the existing open-source DDD methodologies. First, real crystalline materials are elastically anisotropic, and the computation of both stress fields and, moreover, displacement fields of dislocations in anisotropic materials remains challenging. Second, real atomistic dislocations have a character-dependent core energy that may be difficult to represent within continuum DDD models. Here, we have calibrated the edge dislocation bow-out by adjusting the core energy model in ParaDis [6]. Recent work [26] suggests this may be insufficient, and so new models may be needed. The numerical solution of the full coupled boundary value problem remains necessary to solve many problems of interest wherein (i) the atomistic domain contains other defects (cracks, voids, inclusions, etc.), (ii) the boundary conditions are essential to the solution of the problem or (iii) stresses on dislocations in the vicinity of the interface introduce non-negligible spurious forces such that the passing distance becomes impracticably large with our approximate method. We are currently developing an efficient numerical solution based on Greens function methods to address this last issue and will report on our progress in the near future.

\section{Acknowledgements}

The authors thank the Fonds National Suisse for support of this work through the project 200021-140506 entitled "Coupled Atomistic/Discrete-Dislocations in 3d (CADD3d)". MH would also like to thank Dr. Ben Szajewski for useful discussions on ParaDis and Dr. Jaehyun Cho for providing the core templates.

\section{Appendix A. Displacement field for periodic arrays of edge dislocations}

In what follows we seek to find analytical, closed-form expressions for infinite sums. Under certain assumptions infinite sums can be conveniently evaluated via the Residue theorem from complex analysis:

Theorem 1 (Residue theorem). Let $\mathcal{A}$ be an open set containing $\left\{w_{1}, \ldots, w_{p}\right\}$ and let further $g$ be holomorphic in $\mathcal{A} \backslash\left\{w_{1}, \ldots, w_{p}\right\}$. Then

$$
\frac{1}{2 \pi \mathrm{i}} \int_{\partial \mathcal{A}} g(x) \mathrm{d} x=\sum_{k=1}^{p} \operatorname{res}\left(g(x), w_{k}\right),
$$

where for an $n$-th order pole

$$
\operatorname{res}\left(g(x), w_{k}\right)=\frac{1}{(n-1) !} \lim _{x \rightarrow w_{k}} \frac{\partial^{n-1}}{\partial x^{n-1}}\left(\left(x-w_{k}\right)^{n} g(x)\right) .
$$


In order to evaluate infinite sums we multiply the function $g(x)$ with a function $h(x)=\pi \cot \pi x$ and use the fact that $h(x)$ has simple poles $\forall x \in \mathcal{A}=\mathbb{N}$ such that

$$
\frac{1}{2 \pi \mathrm{i}} \int_{\partial \mathcal{A}} g(x) h(x) \mathrm{d} x=\sum_{k=1}^{p} \operatorname{res}\left(g(x) h(x), w_{k}\right)+\sum_{k=-\infty}^{\infty} g(k) .
$$

We assume that the function $g(x)$ goes to zero as $x \rightarrow \infty$. Therefore the left hand side of (A.3) is essentially zero. Thus we have

$$
\sum_{k=-\infty}^{\infty} g(k)=-\sum_{k=1}^{p} \operatorname{res}\left(g(x) h(x), w_{k}\right)
$$

In order to evaluate infinite sums it therefore suffices to determine the poles of $g(x)$ and compute the residues of $g(x) h(x)$.

We consider a periodic array of infinite straight edge dislocations. The separation between the dislocations is $\Delta x_{2}=l_{y}$. The position of the dislocations is thus

$$
\forall i \in \mathbb{N} \quad \boldsymbol{x}_{i}=\left(\begin{array}{c}
0 \\
i \Delta x_{2} \\
0
\end{array}\right)=\left(\begin{array}{c}
0 \\
\Delta x_{2}^{i} \\
0
\end{array}\right)
$$

The isotropic displacement field induced by each dislocation is then given by

$$
\begin{aligned}
& \tilde{u}_{1}^{i}(\boldsymbol{x})=0, \\
& \tilde{u}_{2}^{i}(\boldsymbol{x})=\frac{b}{2 \pi}\left[\frac{\left(x_{2}-\Delta x_{2}^{i}\right) x_{3}}{2(1-\nu)\left(\left(x_{2}-\Delta x_{2}^{i}\right)^{2}+x_{3}^{2}\right)}+\arctan \left(\frac{x_{2}-\Delta x_{2}^{i}}{x_{3}}\right)\right], \\
& \tilde{u}_{3}^{i}(\boldsymbol{x})=-\frac{b}{2 \pi}\left[\frac{\left(x_{2}-\Delta x_{2}^{i}\right)^{2}-x_{3}^{2}}{4(1-\nu)\left(\left(x_{2}-\Delta x_{2}^{i}\right)^{2}+x_{3}^{2}\right)}+\frac{1-2 \nu}{4(1-\nu)} \ln \left(\left(x_{2}-\Delta x_{2}^{i}\right)^{2}+x_{3}^{2}\right)\right] .
\end{aligned}
$$

Henceforth we use the compact notation

$$
\begin{aligned}
& \tilde{u}_{2}^{i}(\boldsymbol{x})=a_{1} f_{11}^{i}(\boldsymbol{x})+b_{1} f_{12}^{i}(\boldsymbol{x}), \\
& \tilde{u}_{3}^{i}(\boldsymbol{x})=a_{2} f_{21}^{i}(\boldsymbol{x})+b_{2} f_{22}^{i}(\boldsymbol{x}),
\end{aligned}
$$

where

$$
a_{1}=\frac{b}{4 \pi(1-\nu)}, \quad a_{2}=-\frac{1}{2} a_{1} \quad b_{1}=\frac{b}{2 \pi}, \quad b_{2}=(1-2 \nu) a_{2}
$$

and

$$
\begin{array}{ll}
f_{11}^{i}(\boldsymbol{x})=\frac{\left(x_{2}-\Delta x_{2}^{i}\right) x_{3}}{\left(x_{2}-\Delta x_{2}^{i}\right)^{2}+x_{3}^{2}}, & f_{12}^{i}(\boldsymbol{x})=\arctan \left(\frac{x_{2}-\Delta x_{2}^{i}}{x_{3}}\right), \\
f_{21}^{i}(\boldsymbol{x})=\frac{\left(x_{2}-\Delta x_{2}^{i}\right)^{2}-x_{3}^{2}}{\left(x_{2}-\Delta x_{2}^{i}\right)^{2}+x_{3}^{2}}, & f_{22}^{i}(\boldsymbol{x})=\ln \left(\left(x_{2}-\Delta x_{2}^{i}\right)^{2}+x_{3}^{2}\right) .
\end{array}
$$

The total displacement field follows by summing the contributions from the individual dislocations, that is

$$
\begin{aligned}
& \tilde{u}_{2}^{\mathrm{PAD}}(\boldsymbol{x})=a_{1} \sum_{i=-\infty}^{+\infty} f_{11}^{i}(\boldsymbol{x})+b_{1} \sum_{i=-\infty}^{+\infty} f_{12}^{i}(\boldsymbol{x}), \\
& \tilde{u}_{3}^{\mathrm{PAD}}(\boldsymbol{x})=a_{2} \sum_{i=-\infty}^{+\infty} f_{21}^{i}(\boldsymbol{x})+b_{2} \sum_{i=-\infty}^{+\infty} f_{22}^{i}(\boldsymbol{x}),
\end{aligned}
$$

It is easy to see that the displacement $\tilde{u}_{3}$ diverges in the second term $f_{22}^{i}$ as $\Delta x_{2}^{i} \rightarrow \infty$. Fortunately, the divergent term is effectively associated with a rigid body shift — since the elastic Green function lacks the definition of boundary conditions. Therefore we can generate a convergent sum using the partial derivatives 
of $f_{22}^{i}(\boldsymbol{x})$ given by

$$
\frac{\partial f_{22}(\boldsymbol{x})}{\partial x_{2}}=\frac{2\left(x_{2}+\Delta x_{2}^{i}\right)}{\left(x_{2}+\Delta x_{2}^{i}\right)+x_{3}^{2}}, \quad \frac{\partial f_{22}(\boldsymbol{x})}{\partial x_{3}}=\frac{2 x_{3}}{\left(x_{2}+\Delta x_{2}^{i}\right)+x_{3}^{2}}
$$

as sums $\sum_{i=-\infty}^{\infty} 1 / r$ converge whereas $\sum_{i=0}^{\infty} 1 / r$ does not. Therefore the infinite sums of the partial derivatives converge.

In the following we generate exemplarily the closed-form solution for the infinite sum $\sum_{i=-\infty}^{+\infty} \partial_{x_{2}} f_{22}(\boldsymbol{x})$. First note that the function $\partial_{x_{2}} f_{22}(\boldsymbol{x})$ has two poles

$$
w_{1}=\frac{-x_{2}+\mathrm{i} x_{3}}{l_{2}}, \quad w_{2}=\frac{-x_{2}-\mathrm{i} x_{3}}{l_{2}} .
$$

Applying the residue theorem we get

$$
\begin{aligned}
\sum_{i=-\infty}^{+\infty} \frac{\partial f_{22}(\boldsymbol{x})}{\partial x_{2}} & =-\sum_{k=1}^{2} \operatorname{res}\left(\partial_{x_{2}} f_{22}^{k}(\boldsymbol{x}) h(k), w_{k}\right) \\
& =C \operatorname{Re}\left(\cot \left[C\left(x_{2}-\mathrm{i} x_{3}\right)\right]+\cot \left[C\left(x_{2}-\mathrm{i} x_{3}\right)\right]\right) \\
& =-\frac{2 C \sin \left(2 C x_{2}\right)}{\left(\cos \left(2 C x_{2}\right)-\cosh \left(2 C x_{3}\right)\right)}
\end{aligned}
$$

with $C=\pi / l_{2}$, since we are only interested in the real part. The infinite sum may then be written as

$$
\sum_{i=-\infty}^{+\infty} f_{22}(\boldsymbol{x}) \equiv \int\left(\sum_{i=-\infty}^{+\infty} \frac{\partial f_{22}(\boldsymbol{x})}{\partial x_{2}}\right) \mathrm{d} x_{2}+I\left(x_{3}\right)<\infty
$$

but $I\left(x_{3}\right)=0$ since

$$
\int \frac{\partial f_{22}(\boldsymbol{x})}{\partial x_{2}} \mathrm{~d} x_{2}=\int \frac{\partial f_{22}(\boldsymbol{x})}{\partial x_{3}} \mathrm{~d} x_{3}
$$

Therefore we have

$$
\sum_{i=-\infty}^{+\infty} f_{22}(\boldsymbol{x}) \equiv \int\left(-\frac{2 C \sin \left(2 C x_{2}\right)}{\left(\cos \left(2 C x_{2}\right)-\cosh \left(2 C x_{3}\right)\right)}\right) \mathrm{d} x_{2}=\ln \left(\left|\cos \left(2 C x_{2}\right)-\cosh \left(2 C x_{3}\right)\right|\right)
$$

To compute the remaining infinite sums we proceed in a similar way. Note that we can apply the residue theorem to $\sum_{i=-\infty}^{+\infty} f_{11}(\boldsymbol{x})$ directly since the series is convergent and consist of rational terms. On the other hand, the sum $\sum_{i=-\infty}^{+\infty} f_{12}(\boldsymbol{x})$ which accounts for the plastic slip is obviously divergent. Therefore we use its partial derivatives as for the logarithmic term in order to omit the rigid body shift. Thus we have

$$
\begin{aligned}
& \sum_{i=-\infty}^{+\infty} f_{11}(\boldsymbol{x}) \equiv-\frac{C x_{3} \sin \left(2 C x_{2}\right)}{\left(\cos \left(2 C x_{2}\right)-\cosh \left(2 C x_{3}\right)\right)}, \\
& \sum_{i=-\infty}^{+\infty} f_{12}(\boldsymbol{x}) \equiv-\arctan \left(\operatorname{coth}\left(C x_{3}\right) \tan \left(C x_{2}\right)\right), \\
& \sum_{i=-\infty}^{+\infty} f_{21}(\boldsymbol{x}) \equiv \frac{2 C x_{3} \sinh \left(2 C x_{3}\right)}{\left(\cos \left(2 C x_{2}\right)-\cosh \left(2 C x_{3}\right)\right)}
\end{aligned}
$$

The closed-form expression of (A.10) therefore reads

$$
\begin{aligned}
& \tilde{u}_{2}^{\mathrm{PAD}}(\boldsymbol{x})=-a_{1} \frac{C x_{3} \sin \left(2 C x_{2}\right)}{\left(\cos \left(2 C x_{2}\right)-\cosh \left(2 C x_{3}\right)\right)}-b_{1} \arctan \left(\operatorname{coth}\left(C x_{3}\right) \tan \left(C x_{2}\right)\right), \\
& \tilde{u}_{3}^{\mathrm{PAD}}(\boldsymbol{x})=a_{2} \frac{2 C x_{3} \sinh \left(2 C x_{3}\right)}{\left(\cos \left(2 C x_{2}\right)-\cosh \left(2 C x_{3}\right)\right)}+b_{2} \ln \left(\left|\cos \left(2 C x_{2}\right)-\cosh \left(2 C x_{3}\right)\right|\right) .
\end{aligned}
$$




\section{Appendix B. Periodic array of curved dislocations}

The previous result can be efficiently used to facilitate the computation of the elastic displacement field of periodic arrays of curved dislocations. Due to the linearity of the problem we can use the analytic, closedform expression (A.20) in order to compute the displacement field of infinite edge dislocations. Subsequently we superimpose the displacement field of the closed loops $\Delta \tilde{\boldsymbol{u}}^{(i, j)}$ for a periodic image $(i, j)$ according to [2, Appendix A 3.4] which account for the bowed components of the dislocations as shown in Figure B.12. Note that we only need to compute the contributions from the surface segments and the solid angle as the inner segments cancel each other out.

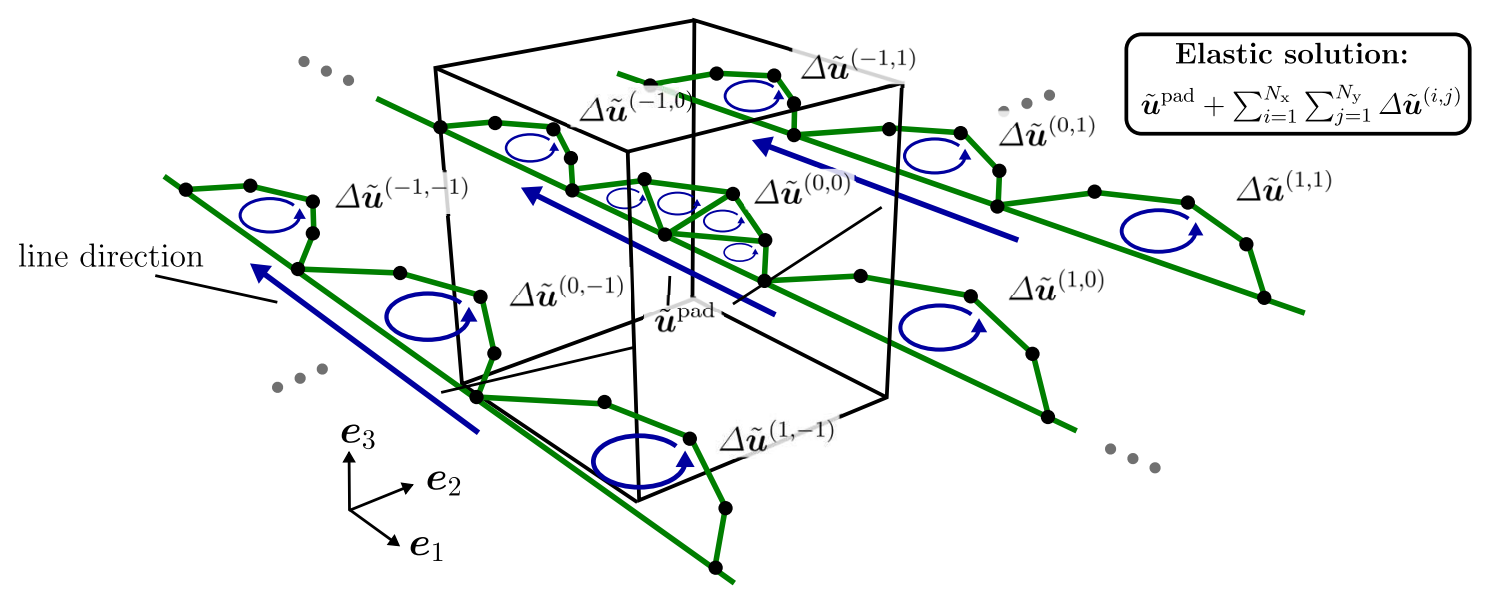

Figure B.12: Schematic illustration of the summation scheme for the computation of the elastic displacement field of periodic arrays of curved dislocations

We further note that the displacement field converges approximately linear in the number of periodic images. Fortunately this is not an issue since we already capture a great portion of the full solution analytically via $\tilde{\boldsymbol{u}}^{\mathrm{PAD}}$. Furthermore our test problem contains only one dislocation. If this scheme should be adopted to larger problems, e.g. to visualize the displacement field in PAD domains containing numerous dislocations, more efficient summation schemes would have to be developed. However, we mainly aim to use this scheme to compute the boundary conditions on the atomistic problem for our specific test case in Section 3.

\section{References}

\section{References}

[1] Amelang, J., Venturini, G., Kochmann, D., 2015. Summation rules for a fully nonlocal energy-based quasicontinuum method. Journal of the Mechanics and Physics of Solids 82, 378-413.

[2] Anciaux, G., Junge, T., Hodapp, M., Cho, J., Molinari, J.-F., Curtin, W., 2017. Coupled atomistic/discrete dislocation method in 3d - part i: Methodology. submitted to Journal of the Mechanics and Physics of Solids.

[3] Arsenlis, A., Cai, W., Tang, M., Rhee, M., Oppelstrup, T., Hommes, G., Pierce, T. G., Bulatov, V. V., 2007. Enabling strain hardening simulations with dislocation dynamics. Modelling and Simulation in Materials Science and Engineering 15 (6), 553.

[4] Barnett, D., 1985. The displacement field of a triangular dislocation loop. Philosophical Magazine A: Physics of Condensed Matter, Structure, Defects and Mechanical Properties 51 (3), 383-387.

[5] Barnett, D., Balluffi, R., 2007. The displacement field of a triangular dislocation loop-a correction with commentary. Philosophical Magazine Letters 87 (12), 943-944.

[6] Cai, W., Arsenlis, A., Weinberger, C., Bulatov, V., 2006. A non-singular continuum theory of dislocations. Journal of the Mechanics and Physics of Solids 54 (3), 561-587. 
[7] Cho, J., Anciaux, G., Curtin, W., Molinari, J.-F., 2017. Coupled atomistic/discrete dislocation method in 3d - part iii: Practical applications. submitted to Journal of the Mechanics and Physics of Solids.

[8] Crone, J. C., Chung, P. W., Leiter, K. W., Knap, J., Aubry, S., Hommes, G., Arsenlis, A., 2014. A multiply parallel implementation of finite element-based discrete dislocation dynamics for arbitrary geometries. Modelling and Simulation in Materials Science and Engineering 22 (3), 035014.

[9] Dewald, M., Curtin, W., 2006. Analysis and minimization of dislocation interactions with atomistic/continuum interfaces. Modelling and Simulation in Materials Science and Engineering 14 (3), 497-514.

[10] Ehrlacher, V., Ortner, C., Shapeev, A. V., 2016. Analysis of boundary conditions for crystal defect atomistic simulations. Archive for Rational Mechanics and Analysis 222 (3), 1217-1268.

[11] Ercolessi, F., Adams, J. B., 1994. Interatomic potentials from first-principles calculations: The forcematching method. EPL (Europhysics Letters) 26 (8), 583.

[12] Hirth, J., Lothe, J., 1982. Theory of Dislocations. Krieger Publishing Company.

[13] Kelchner, C. L., Plimpton, S. J., Hamilton, J. C., Nov 1998. Dislocation nucleation and defect structure during surface indentation. Phys. Rev. B 58, 11085-11088.

[14] Knap, J., Ortiz, M., 2001. An analysis of the quasicontinuum method. Journal of the Mechanics and Physics of Solids 49 (9), 1899-1923.

[15] Kochmann, D., Venturini, G., 2014. A meshless quasicontinuum method based on local maximumentropy interpolation. Modelling and Simulation in Materials Science and Engineering 22 (3).

[16] Miller, R., Shilkrot, L., Curtin, W., 2004. A coupled atomistics and discrete dislocation plasticity simulation of nanoindentation into single crystal thin films. Acta Materialia 52 (2), 271-284.

[17] Mura, T., 1982. Micromechanics of Defects in Solids. Comparative Studies in Overseas History. Springer Netherlands.

[18] Pavia, F., Curtin, W., 2015. Parallel algorithm for multiscale atomistic/continuum simulations using lammps. Modelling and Simulation in Materials Science and Engineering 23 (5).

[19] Plimpton, S., 1995. Fast parallel algorithms for short-range molecular dynamics. Journal of Computational Physics 117 (1), 1-19.

[20] Shenoy, V. B., Kukta, R. V., Phillips, R., Feb 2000. Mesoscopic analysis of structure and strength of dislocation junctions in fcc metals. Phys. Rev. Lett. 84, 1491-1494.

[21] Shilkrot, L., Curtin, W., Miller, R., 2002a. A coupled atomistic/continuum model of defects in solids. Journal of the Mechanics and Physics of Solids 50 (10), 2085-2106.

[22] Shilkrot, L., Miller, R., Curtin, W., 2004. Multiscale plasticity modeling: Coupled atomistics and discrete dislocation mechanics. Journal of the Mechanics and Physics of Solids 52 (4), 755-787.

[23] Shimokawa, T., Mortensen, J., Schiøtz, J., Jacobsen, K., 2004. Matching conditions in the quasicontinuum method: Removal of the error introduced at the interface between the coarse-grained and fully atomistic region. Physical Review B - Condensed Matter and Materials Physics 69 (21), 214104-1$214104-10$.

[24] Stukowski, A., Bulatov, V., Arsenlis, A., 2012. Automated identification and indexing of dislocations in crystal interfaces. Modelling and Simulation in Materials Science and Engineering 20 (8).

[25] Szajewski, B., Curtin, W., 2015. Analysis of spurious image forces in atomistic simulations of dislocations. Modelling and Simulation in Materials Science and Engineering 23 (2). 
[26] Szajewski, B., Pavia, F., Curtin, W., 2015. Robust atomistic calculation of dislocation line tension. Modelling and Simulation in Materials Science and Engineering 23 (8).

[27] Tadmor, E., Ortiz, M., Phillips, R., 1996. Quasicontinuum analysis of defects in solids. Philosophical Magazine A: Physics of Condensed Matter, Structure, Defects and Mechanical Properties 73 (6), 1529 1563.

[28] Weygand, D., Friedman, L., Van Der Giessen, E., Needleman, A., 2002. Aspects of boundary-value problem solutions with three-dimensional dislocation dynamics. Modelling and Simulation in Materials Science and Engineering 10 (4), 437-468.

[29] Xiao, S., Belytschko, T., 2004. A bridging domain method for coupling continua with molecular dynamics. Computer Methods in Applied Mechanics and Engineering 193 (17-20), 1645-1669. 


\section{CADD-3d glossary}

$\Omega \quad$ Continuous body

$\Omega_{\mathrm{A}} \quad$ Continuum domain associated with an atomistic domain

$\Omega_{\mathrm{C}} \quad$ Continuum domain

$\Omega_{\mathrm{P}} \quad$ Pad domain

$\Omega_{\text {core }}$ Dislocation core domain

$\partial \Omega_{\mathrm{I}} \quad$ Artificial sharp interface between the atomistic and the continuum domain

$\boldsymbol{s}_{\text {trans }}$ Transmission node

$\mathcal{P} \quad$ Shorthand notation of a problem definition

$\mathcal{P}^{\mathrm{a}} \quad$ Atomistic problem

$\mathcal{P}^{\mathrm{c}} \quad$ Continuum problem

$\mathcal{P}^{\mathrm{c} / \mathrm{dd}} \quad$ Material subproblem of $\mathcal{P}^{\mathrm{c}}$

$\mathcal{P}^{\mathrm{c} / \mathrm{p}} \quad$ Physical subproblem of $\mathcal{P}^{\mathrm{c}}$

$\mathcal{P}^{\text {cadd }}$ CADD problem

$\tilde{\mathcal{P}}^{\text {cadd }}$ Approximate CADD problem

\section{Atomistic glossary}
$\boldsymbol{r}_{\mathrm{a}}$ Position of an atom
$\boldsymbol{u}_{\mathrm{a}} \quad$ Displacement of $\Omega_{\mathrm{A}}$
$f_{\mathrm{a}} \quad$ Force on an atom in $\Omega_{\mathrm{A}}$
$a_{0} \quad$ Lattice constant
$r_{\mathrm{c}} \quad$ Cut-off radius of the interatomic potential

\section{Dislocation dynamics glossary}

$\gamma \quad$ Discrete dislocation line

$\gamma_{\mathrm{c}} \quad$ Discrete dislocation line in a continuum

$\gamma_{\mathrm{a}} \quad$ Detected discrete dislocation line in an atomistic domain

$\gamma_{\text {hyb }} \quad$ Hybrid discrete dislocation line

$\gamma_{\mathrm{v}} \quad$ Virtual discrete dislocation line

$s \quad$ Material point on a discrete dislocation $\gamma_{\mathrm{c}}$

$\tilde{\boldsymbol{u}} \quad$ Displacement field due to an arbitrarily-shaped closed dislocation in $\mathbb{R}^{3}$

$\tilde{\boldsymbol{u}}_{\text {corr }} \quad$ Template displacement field due to an infinite straight dislocation in $\mathbb{R}^{3}$

$\hat{\boldsymbol{u}} \quad$ Displacement field of the corrective boundary value problem

$U^{\text {core }}$ Total core energy

$W^{\text {core }}$ Core energy term which depends only on local properties of the dislocation line

$f^{\mathrm{pk}} \quad$ Peach-Koehler force

$f^{\text {core }} \quad$ Force on a dislocation line due to $U^{\text {core }}$

$\tilde{\boldsymbol{\sigma}} \quad$ Stress field due to an arbitrarily-shaped closed dislocation in $\mathbb{R}^{3}$

$\hat{\sigma} \quad$ Stress field of the corrective boundary value problem

$\boldsymbol{b}$ Burgers vector

$b \quad$ Absolute value of the Burgers vector

$\vartheta \quad$ Character angle of the dislocation line

a Isotropic core spreading width

$E^{\text {core }} \quad$ Core energy parameter

$\boldsymbol{M}$ Second-order dislocation mobility tensor

$\boldsymbol{v} \quad$ Dislocation velocity vector 


\section{Continuum elasticity}

$\boldsymbol{x} \quad$ Material point in a continuum

$\boldsymbol{u}$ Displacement

$\boldsymbol{u}_{\mathrm{c}} \quad$ Displacement of $\Omega_{\mathrm{C}}$

$U$ Total energy of a mechanical system

$\boldsymbol{\sigma}$ Cauchy stress tensor

$\tau \quad$ Scalar shear stress

$\tau_{\mathrm{PK}} \quad$ Scalar applied shear stress

$\mu \quad$ Shear modulus

$\nu \quad$ Poisson's ratio

\section{General}

$\mathbb{R} \quad$ Set of real numbers

$\mathbb{N} \quad$ Set of natural numbers

$\mathbb{R}^{3} \quad$ Three-dimensional Euclidean space

$f \quad$ Force vector 